\title{
The Redistributive Consequences of Segregation
}

Working Paper 2017 - 12

December 2017

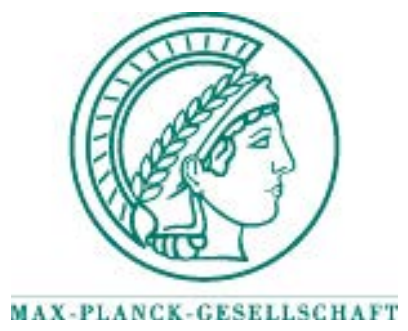

Max Planck Institute for

Tax Law and Public Finance

Department of Business and Tax Law

Department of Public Economics

http:/ / www.tax.mpg.de 
Working papers of the Max Planck Institute for Tax Law and Public Finance Research Paper Series serve to disseminate the research results of work in progress prior to publication to encourage the exchange of ideas and academic debate. Inclusion of a paper in the Research Paper Series does not constitute publication and should not limit publication in any other venue. The preprints published by the Max Planck Institute for Tax Law and Public Finance represent the views of the respective author(s) and not of the Institute as a whole. Copyright remains with the author(s).

Max Planck Institute for Tax Law and Public Finance

Marstallplatz 1

D-80539 Munich

Tel: $\quad+498924246-0$

Fax: $\quad+498924246-501$

E-mail: ssrn@tax.mpg.de

http://www.tax.mpg.de 


\title{
The Redistributive Consequences of Segregation*
}

\author{
Lisa Windsteiger ${ }^{\dagger}$
}

\begin{abstract}
In this paper I present a model in which an increase in inequality can lead to a decrease in voters' demand for redistribution. In my model, people sort into groups according to income and as a result they become biased about the shape of the income distribution. I demonstrate that an increase in inequality can lead to a decrease in perceived inequality in the presence of segregation, and hence to a fall in people's support for redistribution. I motivate my main assumptions with empirical evidence from a small survey that I conducted via Amazon Mechanical Turk.
\end{abstract}

JEL Classification: D31, D83, H24, H53

Keywords: Personal Income Distribution, Inequality, Redistribution

*I thank Ronny Razin, Stephane Wolton, Thomas Piketty, Mike Savage, Matt Levy, Camille Landais, Gilat Levy, Daniel Laurison, Dominik Hangartner, Stephan Maurer, Frank Cowell and the participants of the LSE Microeconomic Theory Work in Progress Seminar for their helpful suggestions and comments.

${ }^{\dagger}$ Max Planck Institute for Tax Law and Public Finance, Email: Lisa.Windsteiger@tax.mpg.de 


\section{Introduction}

Most industrialized countries have seen a remarkable increase in income and wealth inequality over the past 35 to 40 years (see e.g. Piketty (2014)). At the same time, support for redistributive policies hasn't exhibited a comparable trend in the majority of these countries. For instance, demand for redistribution as proxied by realized tax - and redistribution rates has remained relatively constant or even decreased over the last two decades in the US (see e.g. Kuziemko et al. (2015), Ashok et al. (2015) and Piketty et al. (2014)).

There are of course many reasons, above all institutional ones, why realized tax rates need not reflect demand for redistribution well. However, also demand for redistribution as measured by household surveys has not evolved in the same way as (income) inequality (see Ashok et al. (2015) and Kenworthy and McCall (2008)). This is at odds with standard Political Economy models, which predict that high rates of income inequality trigger high demand for redistribution. In the baseline model of Meltzer and Richard (1981) for instance, the redistribution rate that is determined by majority voting is increasing in the difference between median and mean income.

Rising income and wealth inequality have frequently been accompanied by an increase in socio-economic segregation. Watson (2009) and Reardon and Bischoff (2011) demonstrate that both income inequality and income segregation have risen sharply in the US between 1970 and 2000, especially in metropolitan areas. Middle-income neighbourhoods have often made way for both rich and poor communities, and segregation and the erosion of the middle class have gone hand in hand.

In the present paper, I combine these observations with the finding that people tend to misperceive the shape of the income distribution. Empirical studies in the US and Australia find that people underestimate income and wealth inequality and wage differentials (see e.g. Norton and Ariely (2011) and Kiatpongsan and Norton (2014)), and I detect similar types of misperception in my own survey conducted in the US via Amazon Mechanical Turk (see Section 5).

Connecting all these pieces, I build a model that explains why demand for redistribution in society is often lower than predicted by standard Political Economy models (given the prevailing degree of income inequality) and, more importantly, why the relationship between income and wealth inequality and support for redistributive policies will in general be nonmonotone.

In my model, people are segregated according to income, and therefore interact mainly with others who have similar incomes to themselves. As a result, they lose sight of the overall income distribution and become biased 
about the shape of the income distribution. Specifically, they underestimate how different others are to themselves and therefore underestimate income inequality. This has an effect on their support for redistributive policies: People in my model will in general demand less redistribution than in a model without misperceptions. Furthermore, I show that an increase in inequality will, in the presence of segregation and misperceptions, always lead to a smaller increase in demand for redistribution than in a model where people are unbiased, and that it can under certain circumstances even lead to a decrease in demand for redistribution.

At the end of the paper I support my assumptions about misperceptions of the income distribution and segregation by presenting evidence from a survey that I conducted via Amazon Mechanical Turk.

The rest of this paper is organized as follows: Section 2 discusses related literature. Section 3 presents a theoretical model of economic sorting with misperceptions where people underestimate inequality and Section 4 applies this model to the issue of voting for redistribution. Section 5 presents suggestive empirical evidence on misperceptions about the shape of the income distribution and on how socio-economic segregation and misperceptions of the income distribution are related. Section 6 concludes.

\section{Relation to existing literature}

Standard political economy models (see e.g. Meltzer and Richard (1981)) predict that the demand for redistribution should be higher, the poorer the median earner is relative to average income in society. However, studies comparing pre-tax income inequality to redistribution rates in democracies, and hence trying to confirm the Meltzer-Richard Model empirically, deliver mixed results. Some papers do indeed find a positive link between inequality and redistribution (see e.g. Borge and Rattsoe (2004), Meltzer and Richard (1983) and Milanovic (2000)). However, others detect a negative relationship (e.g. Georgiadis and Manning (2012) and Rodriguez (1999)) or no significant link at all (e.g. Kenworthy and McCall (2008) and Scervini (2012)).

There are various explanations for why a high degree of inequality might not be reflected in high realized redistribution rates in an economy: Bartels (2009) argues that the views of the majority might be disregarded by political leaders due to successful lobbying of the financially powerful. Moreover, poor people might participate in the political process to a lesser degree than rich people, which might shift the identity of the median - decisive - voter (see e.g. Larcinese (2005)). Finally, and importantly, people rarely get to vote directly on redistribution rates. Instead, policital candidates offer platforms that take a position on a variety of issues, and people might vote 
against their interest on the subject of redistribution if they consider other issues to be more important (see Matakos and Xefteris (2016)). ${ }^{1}$ However, apart from these factors, which affect realized redistribution rates, it seems to be the case that even the pure redistributive preferences of the population are not in line with what we might call the "Meltzer-RichardHypothesis": that pre-tax inequality and the demand for redistribution should be positively correlated, both across countries and over time (see e.g. Ashok et al. (2015)).

In the Meltzer-Richard Model, people aim to maximize their own aftertax income and hence their sole concern is their relative position in the income distribution as a direct predictor of how much they would benefit or lose from redistribution. More detailed models allow for people's preferences for redistribution to be influenced also by other factors, such as social mobility, the overall degree of inequality in society or social status concerns (see e.g. Piketty (1995), Benabou and Ok (2001), Alesina and Angeletos (2005) and Corneo and Gruener (2000)). This can explain why the median voter's relative position in the income distribution is not necessarily a good predictor of a society's demand for redistribution. However, also in these more elaborate models it will be the case that if inequality increases (ceteris paribus), demand for redistribution increases. ${ }^{2}$ Nevertheless, empirically we find that periods of increasing inequality can be accompanied by stagnant or declining demand for redistribution.

The main contribution of my paper is that I show how my model of endogenous segregation and belief formation can be used to explain a decrease in support for redistribution in societies where inequality increases: As voters interact only with people who have similar income to their own, they underestimate inequality and poor people (including the median voter) underestimate how much they could gain from redistribution. I demonstrate that with endogenous segregation and beliefs, the relationship between redistributive demand and inequality can be non-monotone: an increase in inequality can lead to a decline in demand for redistribution, because people - as they see only a select group of society - might wrongly perceive that inequality has gone down due to the change in the income distribution and endogenous segregation.

There is a growing empirical literature on people's misperceptions of the income distribution. Cruces et al. (2013) find that poor people in Buenos

\footnotetext{
${ }^{1}$ For a concise overview see Bonica et al. (2013).

${ }^{2}$ A notable exception here is Corneo and Gruener (2000), where an increase in economic inequality can lead to a decrease in the preferred tax rate of the middle class due to status concerns - the signalling power of wealth decreases more rapidly with the tax rate if income inequality is high and the middle class want to avoid mixing with the lower class. Note however that this depends crucially on the assumption that social and economic inequality move independently and that the middle class has a higher than average social status and a lower than average economic status.
} 
Aires overestimate their relative position in the income distribution, while rich people underestimate it. They also show that this lowers poor people's demand for redistribution: when their biases are corrected, poor people's demand for redistribution increases. Importantly, they additionally show that (geographical) economic segregation affects people's misperceptions. Karadja et al. (2015) conduct a similar study for Sweden and find that a majority of people there tend to underestimate their relative position. Norton and Ariely (2011) and Norton et al. (2014) find that people in the US and Australia tend to underestimate income and wealth inequality and Kiatpongsan and Norton (2014) find that people underestimate pay differences between different professions.

My own empirical survey described in Section 5 differs from the above, because I focus explicitly on the role of social segregation: In addition to asking people about their perception of the income distribution, I also ask a range of questions concerning people's social circle. Then I construct a variable that proxies the diversity of people's social interactions, and I find that on average people with homogeneous social contacts exhibit more severe misperceptions about the shape of the income distribution than people with heterogeneous social contacts.

\section{Sorting with misperceptions}

In the following section I will introduce a theoretical model of sorting with misperceptions. With the help of this framework I can then predict how groups in society will look like in equilibrium and - because social interactions affect beliefs - also what kind of misperceptions people will have about the overall income distribution. The model below is a simplified version of a more general model presented in Windsteiger (2017a) and can be regarded as an extension to the model of unbiased sorting used in Levy and Razin (2015).

Let income $y$ in an economy be distributed according to an income distribution $F(y)$, on the interval $Y=\left[0, y_{\max }\right]$ where $y_{\max }<\infty$. Assume furthermore that $F(y)$ is continuous and strictly monotonic. As $F(y)$ is an income distribution, I will also assume that $F(y)$ is positively skewed (meaning that the median income is smaller than the average income).

Suppose that an agent's utility is increasing not only in her own income but also in the average income of the people that she interacts with, which I will henceforth call her "reference group". Specifically, a person with income $y_{j}$ obtains utility $U_{j}=y_{j} E\left(y \mid y \in S_{i}\right)$, where $S_{i}$ is individual $j$ 's reference group. If there is no economic segregation, everybody's reference group is a representative sample of the whole population, such that $U_{j}=$ $y_{j} E(y)$. However, a person with income $y_{j}$ can pay a fee $b>0$ to join group 
$S_{b}$ and get utility

$$
y_{j} E\left[y \mid y \in S_{b}\right]-b
$$

or refrain from paying $b$ and get

$$
y_{j} E\left[y \mid y \in S_{0}\right]
$$

where $S_{b}$ is the set of incomes $y$ of people who have paid $b$ and $S_{0}$ is the set of incomes $y$ of people who haven't paid $b$.

Before introducing misperceptions into the model, I want to comment on how to interpret two vital components of this model: the sorting fee $b$ and the supermodular utility function $U$. If we think of sorting and segregation as happening in all realms of life, be it in terms of where we live, where we go on holidays or where we send our kids to school, then the sorting fee refers to the extra sum we need to pay to live in rich neighbourhoods, stay in good hotels and send our kids to posh schools. The utility derived from interacting with people in a certain reference group is increasing in a person's own income and average income of the people she interacts with. If we think about reference groups as social circles and neighbourhoods, then this type of utility function captures for instance the fact that living in an affluent neighbourhood offers many benefits, such as good schools (because people are willing to spend more on the education of their kids, and because the presence of children of rich people might increase other pupil's chances in life through various peer effects) and pleasant surroundings such as parks or leisure centres (perhaps with increased security or surveillance). Instead of modelling all this on a micro level, I subsum all these effects into a utility function which is increasing in the average income of one's peers. The supermodular form of the utility function implies that rich people value (or benefit from) staying with other rich people more than poor people do, which might for instance capture network effects that yield higher benefits the higher one's economic status. ${ }^{3}$

Suppose that people, once they are sorted into their group $S_{b}$ or $S_{0}$, become biased about average income in the other group and hence about the overall income distribution. I will model a group's belief about the other group as resulting from a group belief "technology". Specifically, I will assume that people's biased perception of the other group's average income can be characterized by the continuous belief function

$$
B: \mathbf{P} \rightarrow Y^{4}
$$

where $\mathbf{P}$ is the space of all monotone partitions $P=\left[S_{0}, S_{b}\right]$ of $Y$. For the following analysis, I will restrict my attention to monotone partitions,

\footnotetext{
${ }^{3}$ As I demonstrate below, supermodularity also yields assortative matching in equilibrium which is in line with the matching patterns we observe in real life.
} 
i.e. partitions $P=\left[S_{0}, S_{b}\right]$ of $Y$ that can be uniquely characterized by a cutoff $\hat{y} \in Y$ (with the convention that $S_{0}=[0, \hat{y})$ and $S_{b}=\left[\hat{y}, y_{\max }\right]$ ), and I will henceforth call the people in $S_{0}$ "the poor" and the people in $S_{b}$ "the rich". Without further assumptions, also non-monotone equilibria are possible if people have misperceptions. In Appendix 7.1 I show that restricting attention to monotone partitions is without loss of generality for the analysis that I conduct in this paper. ${ }^{4}$

I will assume that people are correct about average income in their own group. Furthermore, I require misperceptions to be constant within groups, i.e. people who are in the same group have the same misperception about the other group's average (and thus misperceptions do not depend on one's own income directly, but on group membership).

The belief function $B$ is thus a continuous function that maps all monotone partitions of $Y$ (and note that any monotone partition can be uniquely characterized by the cutoff $\hat{y}$ ) into a four-dimensional vector of beliefs

$$
B(\hat{y})=\left(\underline{E}(\hat{y}), \bar{E}_{p}(\hat{y}), \underline{E}_{r}(\hat{y}), \bar{E}(\hat{y})\right)
$$

where the first two entries denote the poor group's belief about average income in the poor and the rich group respectively and the last two entries denote the rich group's belief about average income in the poor and the rich group. $\underline{E}(\hat{y})$ is the true average income in the poor group, i.e. $\underline{E}(\hat{y})=$ $E[y \mid y<\hat{y}]$ and $\bar{E}(\hat{y})$ is the correct average income in the rich group, $\bar{E}(\hat{y})=$ $E[y \mid y \geq \hat{y}]$. The poor's belief about average income in the rich group is $\bar{E}_{p}(\hat{y})$ and the rich's belief about average income in the poor group is $\underline{E}_{r}(\hat{y})$.

Given the belief function $B$, I can define the following:

Definition 1 A monotone partition of $Y$ (characterized by an equilibrium cutoff $\left.\hat{y}^{*}\right)$ and a sorting fee $b>0$ constitute a biased sorting equilibrium iff

$$
\begin{aligned}
y \bar{E}_{p}\left(\hat{y}^{*}\right)-b & <y \underline{E}\left(\hat{y}^{*}\right) \quad \forall y \in\left[0, \hat{y}^{*}\right) \\
y \bar{E}\left(\hat{y}^{*}\right)-b & \geq y \underline{E}_{r}\left(\hat{y}^{*}\right) \quad \forall y \in\left[\hat{y}^{*}, y_{\max }\right]
\end{aligned}
$$

The misperceptions about the other group that people suffer from once they sort into their preferred group affect people's perceived trade-off of choosing one group over the other: People still know exactly what they get if they stay in their own group, but they become biased about what they could get if they would switch to the other group. In a biased sorting equilibrium, people stay in the group that they think gives them the highest utility. Despite the biases that afflict them once they sort into one of the groups, they do not want to switch to the other group. Such an equilibrium

\footnotetext{
${ }^{4}$ I show that the refinement that I introduce in this section (the consistency requirement), implies monotonicity.
} 
is therefore a partition of $Y$ that is "stable" given people's misperceptions about the other group. People compare the utility they obtain in their own group to the utility they think they could obtain in the other group, given their misperceptions about average income in the other group. In a biased sorting equilibrium people think that they reach the highest possible level of utility in their own group and therefore they do not want to move to the other group.

Assuming that people have misperceptions about average income in the other group creates consistency issues: In a biased sorting equilibrium, people's beliefs about the other group can be inconsistent with what they see. A person in the poor group might wonder why a person in the rich group finds it worthwhile to pay $b$, given the poor person's belief about average income in the rich group. Similarly, a person in the rich group might - given the rich group's misperception about average income in the poor group - wonder why a certain person in the poor group doesn't want to join the rich group.

However, this inconsistency vanishes if I introduce what I call the consistency requirement. A partition of society satisfies consistency if people's beliefs about the other group are in line with what they observe: People who are in the poor group think that the people who are in the rich group are correct in doing so and vice versa. Formally, the consistency requirement translates to

Definition 2 A monotone partition of $Y$ (characterized by a cutoff $\hat{y}$ ) and a sorting fee $b$ satisfy consistency iff

$$
\begin{aligned}
& y \bar{E}(\hat{y}))-b \leq y \underline{E}_{r}(\hat{y}) \quad \forall y \in[0, \hat{y}) \\
& \left.y \bar{E}_{p}(\hat{y})-b \geq y \underline{E}(\hat{y})\right) \quad \forall y \in\left[\hat{y}, y_{\max }\right]
\end{aligned}
$$

In words, condition (CR1) requires that a person in the rich group who looks at any person with income $y$ in the poor group thinks that this person cannot achieve higher utility by switching to the rich group (and note that the person from the rich group evaluates person $y$ 's utility in the poor group given her own biased perception of average income in the poor group, $\left.\underline{E}_{r}(\hat{y})\right)$. Condition (CR2) does the same for poor people's belief about the rich group.

Without misperceptions, consistency is implicit in any sorting equilibrium. Because everybody has the same (correct) understanding of average incomes in both groups, people cannot be "puzzled" by other people's choices - everybody evaluates everybody else's utility in the same way. It is only when people have incorrect perceptions of the other group that consistency becomes a separate issue and is not implicit in the equilibrium definition. People can be happy with their own choices (which means the partition constitutes a biased sorting equilibrium), while at the same time 
not understanding other people's choices (which means that consistency is violated). Hence, it makes sense - as a refinement to biased sorting equilibria - to define biased sorting equilibria which additionally satisfy consistency:

Definition 3 A monotone partition of $Y$ (characterized by an equilibrium cutoff $\left.\hat{y}^{*}\right)$ and a sorting fee $b>0$ constitute a biased sorting equilibrium with consistency iff

$$
\begin{aligned}
y \bar{E}_{p}\left(\hat{y}^{*}\right)-b & \leq y \underline{E}\left(\hat{y}^{*}\right) \quad \forall y \in\left[0, \hat{y}^{*}\right) \\
y \bar{E}\left(\hat{y}^{*}\right)-b & \geq y \underline{E}_{r}\left(\hat{y}^{*}\right) \quad \forall y \in\left[\hat{y}^{*}, y_{\max }\right]
\end{aligned}
$$

and

$$
\begin{aligned}
& \left.\left.y \bar{E}\left(\hat{y}^{*}\right)\right)-b \leq y \underline{E}_{r}\left(\hat{y}^{*}\right)\right) \quad \forall y \in\left[0, \hat{y}^{*}\right) \\
& y \bar{E}_{p}\left(\hat{y}^{*}\right)-b \geq y \underline{E}\left(\hat{y}^{*}\right) \quad \forall y \in\left[\hat{y}^{*}, y_{\max }\right]
\end{aligned}
$$

It is immediate to see that these four conditions can be combined to yield the following:

Corollary 1 A monotone partition of $Y$ (characterized by a cutoff $\hat{y}^{*}$ ) and a sorting fee $b>0$ constitute a biased sorting equilibrium with consistency iff

$$
\begin{aligned}
& \hat{y} \bar{E}_{p}\left(\hat{y}^{*}\right)-\hat{y} \underline{E}\left(\hat{y}^{*}\right) \\
= & \hat{y}^{*} \bar{E}\left(\hat{y}^{*}\right)-\hat{y}^{*} \underline{E}_{r}\left(\hat{y}^{*}\right) \\
= & b
\end{aligned}
$$

A biased sorting equilibrium with consistency is thus a partition where the perceived benefit of being in the rich group rather than the poor group (in terms of utility) of the person with income at the equilibrium cutoff $\hat{y}^{*}$ is regarded to be equally high by both groups. Note that for a given equilibrium cutoff $\hat{y}^{*}$ that satisfies (1), the corresponding sorting fee $b$ is unique.

The equilibrium condition (1) restricts the set of belief functions which imply equilibrium existence (see Windsteiger (2017a)). For the remainder of the present paper I want to focus on a particular type of belief function: One where the poor underestimate average income in the rich group and the rich overestimate average income in the poor group, and therefore both groups underestimate income inequality. As I argue in the introduction, this is what empirical evidence shows. I will present suggestive evidence for such misperceptions and how they are connected to segregation in Section 5, where I explain a survey that I have conducted myself via Amazon Mechanical Turk. 
As I demonstrate in the next section, it turns out that with the particular belief function that I use to model people's misperceptions there exists a unique equilibrium partition that satisfies consistency. Without requiring consistency, a range of $\hat{y}$ s would be potential equilibrium cutoffs for this same belief function. Hence, in addition to being a natural refinement concept, consistency can also achieve uniqueness of equilibrium, which in turn makes the comparative statics of Section 4 possible. In Appendix 7.5, I explain the consistency requirement (as applied to the particular belief function used in the next section) in more detail, point out which cutoffs would be equilibria that don't satisfy consistency and under what circumstances the results of Section 4 would still hold in the absence of consistency.

\subsection{Underestimating Inequality}

Suppose the belief function is such that people in the poor group think that average income in the rich group is

$$
\bar{E}_{p}(\hat{y})=\beta(1-F(\hat{y})) \hat{y}+(1-\beta(1-F(\hat{y})) \bar{E}
$$

and people in the rich group think that average income in the poor group is

$$
\underline{E}_{r}(\hat{y})=\gamma F(\hat{y}) \hat{y}+(1-\gamma F(\hat{y})) \underline{E} .
$$

The parameters $\beta \in[0,1]$ and $\gamma \in[0,1]$ describe the "naivity" of agents if $\beta$ (or $\gamma$ ) is 0 , poor (resp. rich) agents have no misperceptions, if $\beta$ (or $\gamma$ ) is 1 people in the respective group are maximally biased for any cutoff $\hat{y}$. It is straightforward to see that $\bar{E}_{p}(\hat{y})<\bar{E}(\hat{y})$ and $\underline{E}_{r}(\hat{y})>\underline{E}(\hat{y})$ for all $y \in\left(0, y_{\max }\right)$, i.e. the poor underestimate average income in the rich group and the rich overestimate average income in the poor group for any interior cutoff.

The functional form of $\bar{E}_{p}(\hat{y})$ and $\underline{E}_{r}(\hat{y})$ implies that the misperceptions are more severe, the smaller the part of the distribution that they can fully observe (which is $F(\hat{y})$ for the poor group and $1-F(\hat{y})$ for the rich group). Specifically, we have that

$$
\frac{d\left(\bar{E}(\hat{y})-\bar{E}_{p}(\hat{y})\right)}{d \hat{y}}=-\beta(1-F(\hat{y}))<0 \quad \forall \hat{y} \in\left(0, y_{\max }\right)
$$

and

$$
\frac{d\left(\underline{E}_{r}(\hat{y})-\underline{E}(\hat{y})\right)}{d \hat{y}}=\gamma F(\hat{y})>0 \quad \forall \hat{y} \in\left(0, y_{\text {max }}\right)
$$

and therefore the misperceptions converge to the truth monotonically as $\hat{y}$ 
goes to 0 resp. $y_{\max } .^{5}$

Misperceptions of this type could arise in the following way: As people live in their segregated communities, they see mostly people who have income similar to their own (i.e. people from their own group). They do sometimes meet people from the other group, but they are not aware that most of the time they do not meet a representative sample of the other group (because they are more likely to meet people from the other group who are close to the cutoff). They see the average income in their own group, but what matters for their sorting decision is also the average income in the other group, which they do not see. Because they know the cutoff $\hat{y}$ and the overall range of $y$ (i.e. that $y$ ranges from 0 to $y_{\max }$ ), they know that the average income of the other group lies somewhere between the cutoff $\hat{y}$ and 0 resp. $y_{\max }$. However, as they neglect the fact that they often do not meet a representative sample of the other group and are rather more likely to meet people very close to the cutoff, the poor think that the average in the rich group is closer to their own average than it actually is, and the same holds for the rich when thinking about the poor group's average. In short, people below the cutoff underestimate average income in the rich group and people above the cutoff overestimate average income in the poor group. This makes both groups underestimate the benefits of sorting: The rich because they think the poor are less poor than they actually are, and the poor because they think the rich are not as rich as they actually are. ${ }^{6}$

The equilibrium cutoff with consistency can be calculated via the equi-

\footnotetext{
${ }^{5}$ For the following analysis it is not necessary that the misperceptions are of exactly of the form (2) and (3). For the results of the next section to hold, I need the misperceptions to be such that a binary biased sorting equilibirum exists and is (ideally) unique. Sufficient conditions for this are stated in Windsteiger (2017a). Furthermore, the equilibrium cutoff needs to be located above median income. In Appendix 7.2, I specify sufficient conditions on the belief function to guarantee that there is a unique interior equilibrium cutoff above the median.

${ }^{6}$ The specific form of misperception that I use in this paper can be microfounded in the following way: People in the poor group only sometimes encounter a representative sample of the rich (e.g. if they go to the opera, watch a royal wedding or shop in a fancy store) and the rest of the time encounter only rich people who are very close to the cutoff (basically at $\hat{y}$ ), maybe because they are parents of their kids' school friends (uppermiddle class families sometimes prefer to send their kids to state schools). However, people are not aware of this and therefore estimate average income as if they were observing a representative sample of the other group. The particular functional form of the bias can arise if the frequency of meeting a representative sample of the other group depends on the size of the own group, $F(\hat{y})$. This could be because "meeting a representative sample" does not actually require personal encounter but also comprises accounts from other people who are in one's own group. Then if people from different groups meet each other at a certain rate, the group with the bigger mass has a better understanding of the other group because people learn from others in their own group.
} 
librium condition

$$
\hat{y}^{*}\left[\bar{E}\left(\hat{y}^{*}\right)-\underline{E}_{r}\left(\hat{y}^{*}\right)\right]=\hat{y}^{*}\left[\bar{E}_{p}\left(\hat{y}^{*}\right)-\underline{E}\left(\hat{y}^{*}\right)\right] .
$$

The expressions on both sides also need to be equal to some $b>0$, which rules out $\hat{y}=0$ as an equilibrium cutoff. Hence, any equilibrium cutoff $\hat{y}^{*}$ must satisfy

$$
\bar{E}\left(\hat{y}^{*}\right)-\underline{E}_{r}\left(\hat{y}^{*}\right)=\bar{E}_{p}\left(\hat{y}^{*}\right)-\underline{E}\left(\hat{y}^{*}\right) .
$$

Plugging in the functional form of the misperceptions, (2) and (3), and rearranging gives

$$
\beta\left(1-F\left(\hat{y}^{*}\right)\right)\left(\bar{E}\left(\hat{y}^{*}\right)-\hat{y}^{*}\right)=\gamma F\left(\hat{y}^{*}\right)\left(\hat{y}^{*}-\underline{E}\left(\hat{y}^{*}\right)\right)
$$

and thus

$$
\hat{y}^{*}=\frac{\beta\left(1-F\left(\hat{y}^{*}\right)\right) \bar{E}\left(\hat{y}^{*}\right)+\gamma F\left(\hat{y}^{*}\right) \underline{E}\left(\hat{y}^{*}\right)}{\beta\left(1-F\left(\hat{y}^{*}\right)\right)+\gamma F\left(\hat{y}^{*}\right)}
$$

which can be rewritten as

$$
\hat{y}^{*}=\frac{a\left(1-F\left(\hat{y}^{*}\right)\right) \bar{E}\left(\hat{y}^{*}\right)+F\left(\hat{y}^{*}\right) \underline{E}\left(\hat{y}^{*}\right)}{a\left(1-F\left(\hat{y}^{*}\right)\right)+F\left(\hat{y}^{*}\right)}
$$

where $a=\beta / \gamma \cdot{ }^{7}$ An equilibrium cutoff $\hat{y}^{*}$ must thus be a fixed point of the function

$$
h(\hat{y})=\frac{a(1-F(\hat{y})) \bar{E}(\hat{y})+F(\hat{y}) \underline{E}(\hat{y})}{a(1-F(\hat{y}))+F(\hat{y})} .
$$

In Appendix 7.3, I prove that the function $h(\hat{y})$ has a unique fixed point and hence that there always exists a unique biased sorting equilibrium cutoff $\hat{y}^{*}$. If $a=1$ (and thus $\beta=\gamma$ ), (6) simplifies to $\hat{y}^{*}=E$ and the unique biased sorting equilibrium is such that the cutoff is exactly at the mean.

Proposition 1 If $\underline{E}_{r}(\hat{y})$ and $\bar{E}_{p}(\hat{y})$ are defined according to (2) and (3), there exists a unique interior biased sorting equilibrium with consistency, and the unique equilibrium cutoff $\hat{y}^{*}$ is the fixed point of $h(\hat{y})=\frac{a(1-F) \bar{E}+F \underline{\underline{E}}}{a(1-F)+F}$ where $a=\beta / \gamma$. If $a=1$, the unique cutoff is at $\hat{y}^{*}=E$.

In the following section I will discuss what happens in this model if people can decide on the (linear) tax- and redistribution rate via majority voting.

\footnotetext{
${ }^{7} a>0$ if both types are assumed to be naive to some degree, i.e. $\beta>0$ and $\gamma>0$. If one of the groups would be fully sophisticated, e.g. $\gamma=0$, while the other group is naive, then consistency couldn't be satisfied for any (interior) cutoff. If both groups are fully sophisticated, i.e. $\beta=\gamma=0$, the model turns into a standard model of unbiased sorting.
} 


\section{Voting for Redistribution}

Economic segregation can exacerbate inequalities in various ways. Schooling is one prominent example: If children living in affluent areas get better education than children from poor neighbourhoods because their local schools are of a better standard due to high local investment, income inequality in the next generation will be amplified. This effect is specifically pronounced in the United States, where school choice is linked to neighbourhood (see e.g. Chetty et al. (2014)). Moreover, having class mates from rich and influential families might not only have the direct effect on education via better quality of schooling, but might also yield benefits later in life through social connections that lead to better jobs and opportunities (see e.g. Savage (2015)).

In this section, I demonstrate that there might be another channel through which segregation can affect economic inequality: Economic segregation, if it leads to misperceptions of the income distribution, can have significant consequences for support for redistribution in society, and hence for (post-tax and post-redistribution) income inequality.

I show that segregation leads poor people to underestimate what they can gain from redistribution and therefore to show less support for redistribution than if they would have perfect knowledge of the income distribution. Moreover, an increase in inequality (in the form of a mean-preserving spread of the income distribution) always leads to a smaller increase in perceived inequality and therefore in the demand for redistribution than if people were unbiased. The reason for this is that people with income below average fully observe the fall of low incomes, but do not fully see the offsetting increase of high incomes. Therefore, they think that average income has decreased. But because people's gains from redistribution depend positively on the difference between their own income and (perceived) average income, and both decrease if people are biased, demand for redistribution increases less than if people are unbiased and know that average income hasn't changed. I show that the increase in inequality can even be such that perceived inequality declines and therefore people's support for redistribution falls.

In the analysis below, I continue to use the functional forms of $\bar{E}_{p}(\hat{y})$ and $\underline{E}_{r}(\hat{y})$ as specified in (2) and (3) of Section 3.1, because this allows me to derive precise results. However, the general flavour of those results would not change if more general specifications of $\bar{E}_{p}(\hat{y})$ and $\underline{E}_{r}(\hat{y})$ were used that satisfy the conditions for existence of a unique equilibrium above the median, given in Appendix 7.2. 


\subsection{Inequality and the demand for redistribution}

In the following section, I analyze people's desired tax and redistribution rates in my model. Specifically, I examine what degree of income redistribution society will decide on via majority voting. For reasons of simplicity, I assume that only linear taxation is available, but all findings below could be reproduced in an analogous way with progressive redistribution. ${ }^{8}$

With linear redistribution, everybody in the economy has to pay a proportional tax $t$ and the government redistributes the proceeds equally among all its citizens afterwards. Hence, a person with pre-tax income of $y_{i}$ has after-tax and after-redistribution income

$$
(1-t) y_{i}+\tau(t) E
$$

where the function $\tau(t) \leq t$ accounts for the fact that there is a deadweight loss of taxation. ${ }^{9}$ Suppose furthermore that people vote to decide on the tax rate, and that they care only about their own post-tax income.

Meltzer and Richard (1981) have examined the relationship between inequality and the demand for redistribution in this model: If people are unbiased about the income distribution, a person with income $y_{i}$ - when voting for the redistribution rate - will simply choose the tax rate $t$ that maximizes her post-tax income

$$
(1-t) y_{i}+\tau(t) E
$$

As preferences are single-peaked in this case, the tax rate determined by majority voting will be the median earner's optimal tax rate given by

$$
\tau^{\prime}\left(t^{*}\right)=\frac{y^{M}}{E}
$$

if $\frac{y^{M}}{E}<1$ and $t^{*}=0$ otherwise (I do not allow for the possibility of negative redistribution, i.e. $t$ has to be positive or 0$)$. As $\tau^{\prime}(t)$ is decreasing in $t$, the decisive voter's optimal tax rate $t^{*}$ is decreasing in the ratio between median and average income.

The ratio $\frac{y^{M}}{E}$ can be regarded as an, albeit rudimentary, measure of the degree of income equality in society. If the ratio is small, this means the difference between median and mean income is large and the income distribution has a large positive skew with a majority of people earning income below average and a few very rich people. Therefore, income equality is

\footnotetext{
${ }^{8}$ This would, however, require making additional assumptions on how people perceive the shape of the income distribution.

${ }^{9}$ Let $\tau($.$) be such that \tau(t)>0 \forall t \in(0,1), \tau(0)=0, \tau^{\prime \prime}(t) \leq 0, \tau(1)=0, \tau^{\prime \prime \prime}(t) \geq 0$ [this guarantees that $\tau^{\prime}(t)$ is convex and hence also $\tau^{\prime-1}$ is convex, given that $\tau^{\prime}$ is decreasing].
} 
low and the demand for redistribution will be high in that case. If, on the other hand, the income distribution is almost symmetric, with most people being middle-class and only a few at the bottom and the top of the distribution, the equality ratio $\frac{y^{M}}{E}$ will be large (i.e. close to 1 ), and demand for redistribution will be low.

To analyze people's preferences for redistribution if they are biased, I need to establish what their perception of average income is: If people would correctly perceive both average income in their group and average income in the other group, they could simply calculate overall average income via the formula

$$
E=F(\hat{y}) \underline{E}(\hat{y})+(1-F(\hat{y})) \bar{E}(\hat{y})
$$

for any cutoff $\hat{y} \cdot{ }^{10}$ However, if there is economic segregation and people are biased, then people misperceive average income in the other group, and hence they mis-estimate overall average income. Specifically, poor people think that average income is

$$
E_{p}(\hat{y})=F(\hat{y}) \underline{E}(\hat{y})+(1-F(\hat{y})) \bar{E}_{p}(\hat{y})<E .
$$

Because they underestimate average income in the rich group,

$$
\bar{E}_{p}(\hat{y})<\bar{E}(\hat{y})
$$

they end up underestimating overall average income. Analogously, rich people overestimate average income,

$$
E_{r}(\hat{y})=F(\hat{y}) \underline{E}_{r}(\hat{y})+(1-F(\hat{y})) \bar{E}(\hat{y})>E .
$$

Let me for simplicity of exposition assume henceforth that rich and poor people are equally naive, i.e. $\beta=\gamma$ (and hence $a=1),{ }^{11}$ and remember that in this case the equilibrium cutoff will always be at average income $E$. This implies that the median earner is in the poor group (because the income distribution is positively skewed) and her preferred tax rate is given by

$$
\tau^{\prime}\left(\tilde{t}^{*}\right)=\frac{y^{M}}{E_{p}(E)}\left(\text { or } \tilde{t}^{*}=0 \text { if } E_{p}(E)<y^{M}\right)
$$

\footnotetext{
${ }^{10}$ Note that I assume that people know the relative size of their respective group, i.e. they know $F(\hat{y})$ and $1-F(\hat{y})$. They also know the range of the distribution and where the cutoff lies. They only misperceive the shape of the distribution function in the other group. With the type of bias that I examine here, their perceived income distribution in the other group is more skewed towards $\hat{y}$ compared to the actual distribution.

${ }^{11}$ The analysis can be done in a similar way for the general case of $\beta \neq \gamma$. In Appendix $7.4 \mathrm{I}$ analyze the relationship between naivety of the poor relative to the rich, $a$, and the equilibrium cutoff and show that the equilibrium cutoff $\hat{y}^{*}$ is increasing in $a$.
} 
$E_{p}$ is smaller than $E$, hence the median earner's perceived degree of equality as measured by $\frac{y^{M}}{E_{p}(E)}$ is higher than without segregation. Therefore, her optimal tax rate is lower in the presence of economic segregation.

Lemma 1 In the model with segregation and misperceptions the median earner's preferred tax rate is lower compared to the model without misperceptions.

For the remainder of this paper, I will assume that the following condition on the income distribution and people's naivity holds:

Assumption 1 The distance between median and mean income is sufficiently high, such that

$$
\frac{E}{E_{r}(E)} \geq \frac{y^{M}}{E_{p}(E)} \cdot 12
$$

The preferred tax rate of the poorest person in the rich group (i.e. the person earning average income $E$ ) is given by

$$
\tau^{\prime}(t)=\frac{E}{E_{r}(E)} .
$$

If the distance between median and mean income is sufficiently high, such that Assumption 1 holds, then this person will demand a lower tax rate than the median earner, and hence the median earner will be the decisive voter. As the median earner wants less redistribution than in the unbiased case, this yields the following

Proposition 2 The tax rate selected by majority voting in a segregated society where people misperceive the shape of the income distribution as described above is lower than in a society without segregation and misperception of the income distribution.

\subsection{The effect of changing inequality on demand for redistribution}

In the following section I analyze what happens to people's (mis)perceptions and the support for redistribution in a segregated society if income inequality increases and how the effects differ compared to a society without segregation.

When analyzing the effect of an increase in inequality, it is important to clearly specify the exact form of this increase in inequality. Some changes in the shape of the income distribution are such that it cannot even be unequivocally decided whether they lead to an increase or decrease in inequality - different measures of inequality might yield different results. However, 
any mean-preserving spread of the income distribution always implies an increase in inequality, irrespective of the measure that is used, because it can be decomposed into (potentially infinitely many) transfers between rich and poor where money is transferred from a relatively poor to a relatively rich person. It therefore increases all measures of inequality that respect the principle of transfers, such as the Gini coefficient or the Theil index (see also Cowell (2000) and Dalton (1920)). ${ }^{13}$ Hence, I will focus on the effect of a mean-preserving spread of the income distribution on group formation and demand for redistribution in the main part of this paper. Other changes of the income distribution are analyzed in Appendix 7.10 and are briefly summarized at the end of this section.

For simplicity, I will focus on mean-preserving spreads that are such that the mass of people below and above the mean remain the same, but mass shifts from the middle towards the endpoints of the distribution, such that median income declines. ${ }^{14}$ Specifically, I will analyze the effect of what I call a monotone mean-preserving spread of the income distribution, which is such that $\bar{E}(\hat{y})$ increases and $\underline{E}(\hat{y})$ decreases for any cutoff $\hat{y}$ (see Windsteiger $(2017 \mathrm{~b})) \cdot{ }^{15} \mathrm{I}$ will also require that the mean-preserving spread is such that $F(E)$ remains unchanged, and I require Assumption 1 to hold before and after the change in inequality. ${ }^{16}$ As this implies that the me-

\footnotetext{
${ }^{13}$ In the income and wealth inequality literature, an inequality measure is generally required to satisfy four properties: anonymity, scale independence, population independence and the principle of transfers. For an extensive discussion of different inequality measures see Cowell (2000).

${ }^{14}$ This implies that the distance between mean and median income increases.

${ }^{15}$ Such a mean-preserving spread can always be constructed if the initial distribution is strictly monotonic. The easiest way is to just transfer mass from the middle of the distribution to the very endpoints of it (i.e. 0 and $y_{\max }$ ) in such a way that the mean doesn't change.

${ }^{16}$ Note that the definition of a general mean-preserving spread of a distribution requires that mass from the middle of the distribution is transferred to the tails in such a way that the mean of the distribution remains constant (see Rothschild and Stiglitz (1970) or Atkinson (1970)). Formally, we say that $G(y)$ is a mean-preserving spread of $F(y)$ if $(1) \int d G(y)=\int d F(y)$ and $(2) \int_{0}^{\hat{y}}[F(y)-G(y)] d y \leq 0 \forall \hat{y} \in Y$ with strict inequality for some $\hat{y}$. It is immediate to see that this definition doesn't imply that $\bar{E}$ increases and $\underline{E}$ decreases for all cutoffs. For instance, suppose we take mass from the interval $[a, b]$ (where $0<a<b<E$ ) and transfer it to the interval $\left[a^{\prime}, b^{\prime}\right]$ (where $a^{\prime}<a$ and $b^{\prime}<b$ ) and do a symmetric shift of mass to the upper tail from an interval above the mean such that the mean stays constant. This transformation would qualify as a mean-preserving spread, but the conditional expectations at any cutoff below $a$ wouldn't change (or in other words, $\int_{0}^{\hat{y}}[F(y)-G(y)] d y=0 \forall \hat{y}<a$ ). We can ensure that the mean-preserving spread increases $\bar{E}$ and decreases $\underline{E}$ for any cutoff (and is therefore what I call "monotone") if we require that for all values of $y$ smaller than $E$, weight shifts downwards to lower values, and for all values of $y$ larger than $E$, weight shifts upwards to higher values. Formally, this would mean that $F(E)=G(E)$ and that $F$
} 
dian earner is always the median voter, I will use these two expressions interchangeably.

In the absence of segregation and misperceptions, the median voter's support for redistribution increases due to a mean-preserving spread of the above described form, because median income declines relative to average income and hence the equality ratio $\frac{y^{M}}{E}$ decreases,

$$
\Delta\left(\frac{y^{M}}{E}\right)=\frac{\Delta y^{M}}{E}=\frac{\Delta y^{M}}{y^{M}} \frac{y^{M}}{E},
$$

i.e. the percentage change in $\frac{y^{M}}{E}$ is $\frac{\Delta y^{M}}{y^{M}}$ (where $\Delta y^{M}<0$ ). This means that demand for redistribution, given by

$$
\tau^{\prime}\left(t^{*}\right)=\left(\frac{y^{M}}{E}\right)
$$

increases. The increase in the median voter's optimal tax rate $t^{*}$ is

$$
\Delta t^{*}=\tau^{\prime-1}\left(\frac{y^{M}+\Delta y^{M}}{E}\right)-\tau^{\prime-1}\left(\frac{y^{M}}{E}\right) .
$$

In a segregated society, where people misperceive the shape of the income distribution, the effect of an increase in inequality on the support for redistribution depends in general on its impact on the location of the equilibrium cutoff $\hat{y}^{*}$, because this determines people's beliefs about the other group's average income. However, as the equilibrium cutoff $\hat{y}^{*}$ is the fixed point of the function

$$
h(\hat{y})=\frac{a\left(1-F\left(\hat{y}^{*}\right)\right) \bar{E}\left(\hat{y}^{*}\right)+F\left(\hat{y}^{*}\right) \underline{E}\left(\hat{y}^{*}\right)}{a\left(1-F\left(\hat{y}^{*}\right)\right)+F\left(\hat{y}^{*}\right)},
$$

which is at average income $E$ if $a=1$, the position of the equilibrium cutoff does not change due to a mean-preserving spread. ${ }^{17}$

What happens to perceived inequality and the demand for redistribution? As I explained in the previous section, if people are biased due to segregation, the median voter's optimal tax rate $\tilde{t}^{*}$ is characterized by the equation

$$
\tau^{\prime}\left(\tilde{t}^{*}\right)=\left(\frac{y^{M}}{E_{p}(E)}\right)
$$

and $G$ intersect only once, where $F$ cuts $G$ from below ("single-crossing"), and instead of $\int_{0}^{\hat{y}}[F(y)-G(y)] d y \leq 0 \forall \hat{y} \in Y$ we require $\int_{0}^{\hat{y}}[F(y)-G(y)] d y<0 \forall \hat{y} \in\left(0, y_{\max }\right)$.

${ }^{17}$ See Appendix 7.10 for an analysis of general changes of the income distribution, which affect also the location of the equilibrium cutoff. 
where $\tilde{t}^{*}<t^{*}$ (because $E_{p}<E$ ) - the median earner's preferred tax rate is lower under segregation because perceived equality $\frac{y^{M}}{E_{p}}$ is higher.

While average income $E$ does not change due to a mean-preserving spread, I show in Appendix 7.8 that the poor group's average perceived income, $E_{p}$, declines. The poor feel that average income declines because they experience the decline of average income in their own group fully, but only partially take note of the compensating increase in average income among the rich. Hence, they think that society as a whole has become poorer. As a result, the change in the perceived equality ratio $\frac{y^{M}}{E_{p}}$ amounts to

$$
\Delta\left(\frac{y^{M}}{E_{p}}\right)=\frac{\Delta y^{M} E_{p}-y^{M} \Delta E_{p}}{\left(E_{p}\right)^{2}}=\left(\frac{\Delta y^{M}}{y^{M}}-\frac{\Delta E_{p}}{E_{p}}\right) \frac{y^{M}}{E_{p}}
$$

and thus the percentage decrease in $\frac{y^{M}}{E_{p}}$ is $\frac{\Delta y^{M}}{y^{M}}-\frac{\Delta E_{p}}{E_{p}}$, which is smaller (in absolute terms) than the percentage decrease of $\frac{y^{M}}{E}$ in the unbiased case, because $\frac{\Delta E_{p}}{E_{p}}<0$.

Proposition 3 If society is segregated, an increase in inequality (in the form of a monotone mean-preserving spread that keeps $F(E)$ constant) always leads to a smaller percentage increase in the median voter's perceived inequality than in the absence of segregation and misperception.

Moreover, in Appendix 7.8 I demonstrate that one can always construct a mean-preserving spread that leads the median voter to believe that society has become more rather than less equal, i.e. that inequality has decreased rather than increased.

Proposition 4 There exists an increase in inequality that causes a decrease of the median earner's perceived degree of inequality under segregation.

The intuition for Proposition 4 is that, unlike in the non-segregated case, the median voter's perceived equality ratio $\frac{y^{M}}{E_{p}}$ can increase due to a mean preserving spread if people are biased, because both $y^{M}$ and $E_{p}$ decline. If the mean-preserving spread is such that the median voter's perceived degree of inequality decreases, as in Proposition 4, then also the median voter's demand for redistribution (i.e. her preferred tax rate) must necessarily decrease.

Corollary 2 There always exists an increase in inequality such that the tax rate determined by majority voting decreases under segregation.

In Appendix 7.8, I derive the condition on the mean-preserving spread that guarantees Proposition 4. As I explain above, this condition must 
ensure that the decline in $E_{p}$ is larger than the decline in $y^{M}$. I also derive a weaker condition on the mean-preserving spread that guarantees that even if perceived inequality does not decrease, demand for redistribution increases less under segregation than without segregation. The step-by-step calculations in Appendix 7.8 can be summarized as follows: If perceived equality decreases due to a mean-preserving spread under segregation, the fact that the percentage decrease in perceived equality is smaller if society is segregated is not enough to guarantee that also the increase in demand for redistribution will be smaller than without segregation. There are two reasons for this: First, as perceived equality is higher to start with under segregation, a smaller percentage decrease does not automatically imply a smaller absolute decrease than in the absence of segregation. Second, even if the decrease in perceived equality is lower also in absolute terms, it is not clear whether the increase in demand for redistribution will be lower as well: this depends on the shape of the deadweight loss function $\tau($.$) .$ However, it turns out that the assumption that $\tau^{\prime}$ is decreasing and convex is sufficient to ensure that demand for redistribution increases less under segregation if the absolute decrease in perceived equality is smaller than in the absence of segregation. The condition on the mean-preserving spread that guarantees that demand for redistribution under segregation increases by less if inequality increases compared to a situation without segregation is weaker than the condition that is needed for Proposition 4.

In Appendix 7.10, I describe how more general changes in the shape of the income distribution affect demand for redistribution if society is segregated. Specifically, I show that the above described situation of increasing inequality accompanied by declining demand for redistribution will be even more likely to occur if the income distribution changes such that the increase in $\bar{E}$ is not enough to offset the decrease in $\underline{E}$ and hence average income declines. Given that I do not model growth in my model, with uniform growth such a situation would translate into an increase in $\bar{E}$ while $\underline{E}$ stays constant, which is what many argue has happened in advanced economies like the US in the past 20 or 30 years (see e.g. Stiglitz (2015)).

\subsection{Inequality and the supply side of sorting}

An alternative way to model the decline in perceived inequality after an increase in inequality is to assume that there is no segregation in place before the change (because whoever offers the sorting technology doesn't find it worthwhile) but then as inequality increases, offering the sorting technology becomes profitable and therefore society becomes segregated (and people become biased). I examine this in the following section for the case of a profit-maximizing monopolist.

Suppose a profit-maximizing monopolist, who has a fixed cost $c>0$ of 
offering the sorting technology, can decide whether or not to become active. Her profits from offering sorting are

$$
\Pi\left(\hat{y}^{*}\right)=\hat{y}^{*}\left(\bar{E}\left(\hat{y}^{*}\right)-\underline{E}_{r}\left(\hat{y}^{*}\right)\right)\left(1-F\left(\hat{y}^{*}\right)\right)-c
$$

Given that the equilibrium cutoff is at $E$ and substituting for $\underline{E}_{r}$, this can be rewritten as

$$
\Pi(E)=E(E-\underline{E}(E))[1-\gamma F(E)(1-F(E))]-c
$$

Suppose that initially the income distribution is such that

$$
E(E-\underline{E}(E))[1-\gamma F(E)(1-F(E))]-c<0
$$

and hence the monopolist prefers to stay out of the market. If inequality increases (again in the sense of a monotone mean-preserving spread of the income distribution which leaves $F(E)$ constant), $E-\underline{E}$ increases. This means that if the increase in inequality is sufficiently large, the profits from offering the sorting technology will become positive and the society will become segregated. Thus, a large enough increase in inequality will lead to economic segregation.

Lemma 2 Suppose that the income distribution is initially such that a profit maximizing monopolist with fixed costs $c>0$ does not find it profitable to offer the sorting technology. Then for any $c>0$ there exists a mean-preserving spread of the income distribution such that the monopolist's profits become positive.

Hence, I can compare the effect of increasing inequality in the presence of segregation to its effect without taking into account segregation (and the resulting misperception). As in the previous sections, I require Assumption 1 to be satisfied after the increase in inequality, to ensure that the median earner is the decisive voter.

If inequality increases and there is no segregation and people are unbiased, the median voter will demand more redistribution than before the change, because median income $y^{M}$ is smaller as a result of the meanpreserving spread, and hence also $\frac{y^{M}}{E}$ decreases:

$$
\Delta\left(\frac{y^{M}}{E}\right)=\frac{\Delta y^{M}}{E}<0
$$

Therefore, the median earner's demand for redistribution increases from

$$
\tau^{\prime-1}\left(\frac{y^{M}}{E}\right)
$$


to

$$
\tau^{\prime-1}\left(\frac{\dot{y}^{M}}{E}\right)
$$

where $\dot{y}^{M}=y^{M}+\Delta y^{M}<y^{M}$ is median income after the increase in inequality.

If the increase in inequality leads to economic segregation and hence causes people to be biased, then the median voter's demand for redistribution changes from

$$
\tau^{\prime-1}\left(\frac{y^{M}}{E}\right)
$$

to

$$
\tau^{\prime-1}\left(\frac{\dot{y}^{M}}{E_{p}(E)}\right)
$$

where

$$
E_{p}(E)=E-\beta(1-F(E))^{2}(\bar{E}(E)+\Delta \bar{E}(E)-E) .
$$

As $E_{p}<E$, the increase in the median voter's demand for redistribution will be smaller than in the absence of economic segregation.

Proposition 5 If an increase in inequality leads to economic segregation, the median voter's demand for redistribution will increase less than in the absence of segregation.

In Appendix 7.9 I show that I can always construct a mean preserving spread of the income distribution such that demand for redistribution decreases under segregation.

Proposition 6 There exists an increase in inequality that causes economic segregation and leads to a decline in the tax rate determined by majority voting.

Apart from the mean-preserving spread described above there are also other types of increases in inequality that would make it profitable for the monopolist to offer one cutoff. I demonstrate in Appendix 7.6 that for the lognormal distribution an increase in the log-variance $\sigma$ (which corresponds to an increase in the Gini-coefficient but is a median-preserving instead of a mean-preserving spread) also leads to an increase in the monopolist's profits (7).

\section{Empirical Evidence}

In February 2016, I conducted an online survey on 600 US citizens above the age of 18. The survey was distributed via Amazon Mechanical Turk and the 


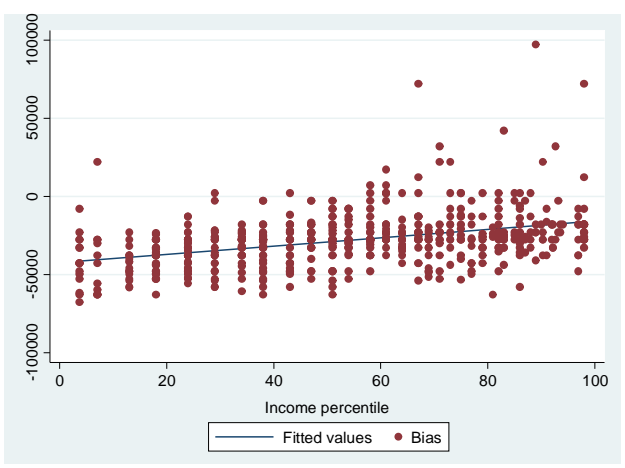

Figure 1: People's estimate of average income is increasing in their own income (Bias = correct average income - perceived average income)

original questionnaire can be accessed at https://1se.ut1.qualtrics . com/jfe/form/SV_eDLNkeGfQg2ycM5. A description of the sample (i.e. respondents' characteristics) can be found in Appendix 8. ${ }^{18}$ The advantages and potential pitfalls of using Amazon Mechanical Turk in academic research have been discussed by Kuziemko et al. (2015) in their Online Appendix. I summarize some of their points and document my own experiences in Appendix 8.1.

By conducting this survey, I wanted to address two main questions:

1. Is there evidence that people misperceive the income distribution in the way I assume in the application of my theoretical model of sorting with misperceptions to the question of demand for redistribution? For example, do poor people underestimate overall average income and do rich people overestimate it?

2. Are people with a diverse social circle (i.e. people who are not very "segregated") less biased?

To tackle the first question, I asked people about their own household income and their estimate of average US household income. Figure 1 plots the relationship between the two: It turns out that, in general, both rich and poor people underestimate mean household income on average. However, people's estimate of average household income is increasing in their own income. This is roughly in line with my model, which would predict that poor people underestimate average income (because they know average income in their group and underestimate average income in the rich group) and rich people overestimate average income (because they know their own average income and overestimate the poor group's income).

The first attempt to identify a link between segregation and misperception is to look at the relationship between the degree of income segregation

\footnotetext{
${ }^{18}$ The data and all do-files are available upon request.
} 
that a respondent lives in and (the absolute value of) her bias. For this purpose, I match the survey data with county-level income segregation data computed by Chetty et al. (2014). However, I do not find any relationship between county-level income segregation and a respondent's absolute level of bias. I suspect that county-level data is too coarse to be useful as a proxy for an individual's degree of segregation. Unfortunately, I cannot repeat the analysis with a more precise measure of income segregation because I have neither lower-level locational information about my respondents, nor data on lower-level income segregation in the US.

However, I also tried to elicit respondents' individual degrees of segregation by asking about the diversity of their social interactions. In particular, I asked them about their friends and colleagues, and how many of them have similar respectively different levels of household income and education. Then I employed a scale from 0 to 4 to classify respondents as more or less segregated (4 indicating the highest possible degree of segregation) concerning those social circles, depending on how similar their work colleagues respectively friends are to themselves. Subsequently, I used factor analysis to identify a common factor out of these categorical response variables (for detailed explanations see Appendix 8).

I find that the severity of misperception of average income is correlated with the degree of social segregation: poor people tend to underestimate average household income less and rich people tend to overestimate it less if their social circle is more diverse. Table 1 shows the results of regressing people's bias about average income (in relative terms) ${ }^{19}$ on their own income percentile, the degree of social segregation as measured by common factor identified by factor analysis and the interaction between own income percentile and the factor: Misperceptions of average household income are less severe for respondents with more diverse social circles.

Furthermore, I asked the so-called "Lin position generator" question in the version of the "Great British Class calculator"20, which is the short version of a similar question asked in the Great British Class Survey (see Savage (2015)). ${ }^{21}$ This question tries to identify the diversity of the respondent's social circle by asking whether she socially knows people with certain occupations (eighteen different occupations), ranging from chief executive to cleaner. I measure diversity of the social circle by assigning to each of the occupations their status rank using the Cambridge Social Interaction and Stratification (CAMSIS) scale score (where low numbers correspond to high rank) and then calculating for each respondent the standard devi-

\footnotetext{
${ }^{19}$ The variable Bias is the difference between the respondent's estimate of average income and true average income as a fraction of true average income. A positive value of Bias thus means average income is overestimated.

${ }^{20}$ see http://www. bbc.co.uk/news/magazine-22000973

${ }^{21}$ The question is named after the sociologist Nan Lin who developed it in the 1980s.
} 
Table 1: Regression results for social segregation as measured by factor analysis

\begin{tabular}{lc}
\hline \hline & Bias \\
\hline Income percentile & 0.0044 \\
& $(0.0006)$ \\
(Income percentile) x (Social segregation) & 0.0024 \\
& $(0.001)$ \\
Social segregation & -0.073 \\
& $(0.060)$ \\
Intercept & -0.598 \\
& $(0.041)$ \\
\hline \hline Standard errors in parentheses & 592 \\
\hline
\end{tabular}

ation of all the scores of occupations she knows: the higher this standard deviation, the more diverse can the respondent's social circle be assumed to be. Regressing the absolute value of people's misperception of average income in relative terms (variable Bias2) on the standard deviation yields significant results and the coefficient has the expected sign: A more diverse social circle corresponds to less bias about average household income (see Table 2).

Table 2: Regression results for social diversity as measured by CAMSIS score standard deviation

\begin{tabular}{lcc}
\hline \hline & $(1)$ & $(2)$ \\
& Bias2 & Bias2 \\
\hline Social circle status diversity & -0.0107 & -0.00916 \\
& $(0.0037)$ & $(0.0037)$ \\
Income percentile & & \\
& & -0.00181 \\
& & $(0.0005)$ \\
Intercept & 0.483 & 0.568 \\
& $(0.031)$ & $(0.0388)$ \\
\hline$N$ & 591 & 591 \\
\hline \hline Standard errors in parentheses & &
\end{tabular}




\section{Conclusion}

In the present paper I have showed how the model of sorting in the presence of misperceptions that is analyzed in detail in Windsteiger (2017a) can be applied to the issue of income inequality and preferences for redistribution: If people are segregated according to income, there will be less demand for redistribution in society. Furthermore, an increase in inequality will lead to a smaller increase in support for redistribution than in the absence of segregation, and certain mean-preserving spreads of the income distribution can even lead to a decrease in demand for redistribution, because they result in a decline in perceived inequality.

Finally, I have reported some of my empirical findings on misperception of the shape of the income distribution and segregation: I have showed evidence that people's estimate of average household income is increasing in their own income, and that people's misperceptions are more severe, the more socially segregated they are.

My approach shows that modelling segregation and belief formation simultaneously can yield interesting and unexpected results and offers new perspectives on issues such as income inequality and redistribution. In the present paper, I have used the model to examine the implications of segregation and biased beliefs on redistributive demand, but the general framework presented in Windsteiger (2017a) offers itself to a wide set of applications related to segregation, such as education policy and housing.

\section{References}

[1] Abdulkadiroğlu, A., Angrist, J., \& Pathak, P. (2014). The elite illusion: Achievement effects at Boston and New York exam schools. Econometrica, 82(1), 137-196.

[2] Alesina, A., \& Angeletos, G. M. (2005). Fairness and Redistribution. American Economic Review, 960-980.

[3] Algan, Y., Do, Q. A., Dalvit, N., Le Chapelain, A., \& Zenou, Y. (2015). How Social Networks Shape Our Beliefs: A Natural Experiment among Future French Politicians. (mimeo)

[4] Andreasch, M., Fessler, P., Mooslechner, P., Schürz, M. (2013) Fakten zur Vermögensverteilung in Österreich, BMASK (Ed.), Sozialbericht 2011-2012 (Vienna 2013) 247-265.

[5] Ashok, V., Kuziemko, I., \& Washington, E. (2015). Support for Redistribution in an Age of Rising Inequality:: New stylized facts and some tentative explanations. Brookings papers on economic activity, (1), 2. 
[6] Bartels, L. M. (2009). Economic inequality and political representation. The unsustainable American state, 167-96.

[7] Becker, G. S. (1974). A theory of marriage. In Economics of the family: Marriage, children, and human capital (pp. 299-351). University of Chicago Press.

[8] Benabou, R., \& Ok, E. A. (2001). Social Mobility and the Demand for Redistribution: The Poum Hypothesis. The Quarterly Journal of Economics, 116(2), 447-487.

[9] Boisjoly, J., Duncan, G. J., Kremer, M., Levy, D. M., \& Eccles, J. (2006). Empathy or antipathy? The impact of diversity. The American economic review, 96(5), 1890-1905.

[10] Bonica, A., McCarty, N., Poole, K. T., \& Rosenthal, H. (2013). Why hasn't democracy slowed rising inequality?. The Journal of Economic Perspectives, 27(3), 103-123.

[11] Borge, L., \& Rattsoe, E. J. (2004). Income Distribution and Tax Structure: Empirical Test of the Meltzer Richard Hypothesis. European Economic Review, 48, 805-826.

[12] Burns, J., Corno, L., \& La Ferrara, E. (2013). Does interaction affect racial prejudice and cooperation? Evidence from randomly assigned peers in South Africa. (mimeo)

[13] Chetty, R., Hendren, N., Kline, P., \& Saez, E. (2014). Where is the land of opportunity? The geography of intergenerational mobility in the United States (No. w19843).

[14] Corneo, G., \& Grüner, H. P. (2000). Social limits to redistribution. The American Economic Review, 90(5), 1491-1507.

[15] Cowell, F. A. (2000). Measurement of inequality. Handbook of income distribution, 1, 87-166.

[16] Cowell, F. (2011). Measuring inequality. OUP Oxford.

[17] Cruces, G., Perez-Truglia, R., \& Tetaz, M. (2013). Biased perceptions of income distribution and preferences for redistribution: Evidence from a survey experiment. Journal of Public Economics, 98, 100-112.

[18] Dalton, H. (1920). The measurement of the inequality of incomes. The Economic Journal, 30(119), 348-361.

[19] Damiano, E., \& Li, H. (2007). Price discrimination and efficient matching. Economic Theory, 30(2), 243-263. 
[20] Dustmann, C., \& Preston, I. (2001). Attitudes to ethnic minorities, ethnic context and location decisions. The Economic Journal, 111(470), 353-373.

[21] Evans, G. \& Tilley, J. (2011). Private schools and public divisions: The influence of private schooling on social attitudes and political choices. in Alison Park et al (eds.), British Social Attitudes: The 28th Report. London: Sage.

[22] Forman, B., \& Koch, C. (2012). Geographic Segregation: The Role of Income Inequality. Communities and Banking.

[23] Georgiadis, A., \& Manning, A. (2012). Spend it like Beckham? Inequality and redistribution in the UK, 1983-2004. Public choice, 151(34), 537-563.

[24] Golub, B., \& Jackson, M. O. (2012). How Homophily Affects the Speed of Learning and Best-Response Dynamics. The Quarterly Journal of Economics, 127(3), 1287-1338.

[25] Gouveia, M., \& Masia, N. A. (1998). Does the median voter model explain the size of government?: Evidence from the states. Public Choice, 97(1-2), 159-177.

[26] Karadja, M., Mollerstrom, J., \& Seim, D. (2014). Richer (and Holier) than Thou? The Effect of Relative Income Improvements on Demand for Redistribution (No. 1050). George Mason University, Interdisciplinary Center for Economic Science.

[27] Kenworthy, L., \& McCall, L. (2008). Inequality, public opinion and redistribution. Socio-Economic Review, 6(1), 35-68.

[28] Kiatpongsan, S., \& Norton, M. I. (2014). How much (more) should CEOs make? A universal desire for more equal pay. Perspectives on Psychological Science, 9(6), 587-593.

[29] Kuziemko, I., Norton, M. I., Saez, E., \& Stantcheva, S. (2015). How elastic are preferences for redistribution? Evidence from randomized survey experiments. The American Economic Review, 105(4), 14781508.

[30] Larcinese, V. (2005). Electoral competition and redistribution with rationally informed voters. Contributions in Economic Analysis \& Policy, $4(1)$.

[31] Levy, G. and Razin, R. (2016). The Coevolution of Segregation, Polarised Beliefs and Discrimination: The Case of Private vs. State Education (mimeo) 
[32] Levy, G., \& Razin, R. (2015). Preferences over equality in the presence of costly income sorting. American Economic Journal: Microeconomics, $7(2)$, 308-337.

[33] Matakos, K. \& Xefteris, D. (2016) Divide and Rule: Redistribution in a Model with Differentiated Candidates. forthcoming in Economic Theory

[34] Meltzer, A. H., \& Richard, S. F. (1981). A rational theory of the size of government. The Journal of Political Economy, 914-927.

[35] Meltzer, A. H., \& Richard, S. F. (1983). Tests of a rational theory of the size of government. Public Choice, 41(3), 403-418.

[36] Milanovic, B. (2000). The median-voter hypothesis, income inequality, and income redistribution: an empirical test with the required data. European Journal of Political Economy, 16(3), 367-410.

[37] Norton, M. I., \& Ariely, D. (2011). Building a better America-One wealth quintile at a time. Perspectives on Psychological Science, 6(1), $9-12$.

[38] Norton, M. I., Neal, D. T., Govan, C. L., Ariely, D., \& Holland, E. (2014). The Not-So-Common-Wealth of Australia: Evidence for a Cross-Cultural Desire for a More Equal Distribution of Wealth. Analyses of Social Issues and Public Policy, 14(1), 339-351.

[39] Pesendorfer, W. (1995). Design innovation and fashion cycles. The American Economic Review, 771-792.

[40] Piketty, T. (1995). Social mobility and redistributive politics. The Quarterly journal of economics, 551-584.

[41] Piketty, T. (2014). Capital in the Twenty-first Century. Cambridge Massachusetts: Belknap of Harvard University Press

[42] Piketty, T., Saez, E., \& Stantcheva, S. (2014). Optimal taxation of top labor incomes: A tale of three elasticities. American Economic Journal: Economic Policy, 6(1), 230-271.

[43] Rayo, L. (2013). Monopolistic Signal Provision. The BE Journal of Theoretical Economics, 13(1), 27-58.

[44] Reardon, S. F., \& Bischoff, K. (2011). Income Inequality and Income Segregation. American Journal of Sociology, 116(4), 1092-1153.

[45] Rodriguez, F. (1999). Inequality, redistribution and rent-seeking. Department of Economics, University of Maryland, 1999b.(mimeo). 
[46] Saez, E., \& Zucman, G. (2016). Wealth Inequality in the United States since 1913: Evidence from Capitalized Income Tax Data. The Quarterly Journal of Economics, 131(2), 519-578.

[47] Savage, M. (2015). Social class in the 21st century. Penguin UK.

[48] Scervini, F. (2012). Empirics of the median voter: democracy, redistribution and the role of the middle class. The Journal of Economic Inequality, 10(4), 529-550.

[49] Stiglitz, J. E. (2015). 8. Inequality and Economic Growth. The Political Quarterly, 86(S1), 134-155.

[50] Watson, T. (2009). Inequality and the measurement of residential segregation by income in American neighborhoods. Review of Income and Wealth, 55(3), 820-844.

[51] Wenglinsky, H. (2007). Are private high schools better academically than public high schools?[electronic resource]. Center on Education Policy, 2007.

[52] Windsteiger, L. (2017a). Sorting in the Presence of Misperceptions. (mimeo).

[53] Windsteiger, L. (2017b). Monopolistic Supply of Sorting, Inequality and Welfare. (mimeo)

\section{Appendix A: Theoretical Appendix}

\subsection{Consistency and monotonicity}

Without imposing the consistency requirement, also non-monotone partitions can be biased sorting equilibria (if the belief function is of a certain form): Suppose that $y_{1} \in S_{b}$ and $y_{2} \in S_{0}$ with $y_{1}<y_{2}$. In order for the partition $\left[S_{0}, S_{b}\right]$ to constitute a biased sorting equilibrium, it must be the case that

$$
y_{1} E_{b}\left[S_{0}\right] \leq y_{1} E\left[S_{b}\right]-b
$$

and

$$
y_{2} E\left[S_{0}\right] \geq y_{2} E_{0}\left[S_{b}\right]-b .
$$

(Notation: $E_{i}\left[S_{j}\right]$ is group $S_{i}$ 's belief about average income in $S_{j}$.) Combined, these two conditions give

$$
y_{2} E_{0}\left[S_{b}\right]-y_{2} E\left[S_{0}\right] \leq b \leq y_{1} E\left[S_{b}\right]-y_{1} E_{b}\left[S_{0}\right]
$$


It is immediate to see that whether this inequality can hold depends on the belief function, because even though $y_{1}<y_{2}$, the misperceptions $E_{0}\left[S_{b}\right]$ and $E_{b}\left[S_{0}\right]$ could be defined in such a way that

$$
y_{1} E\left[S_{b}\right]-y_{1} E_{b}\left[S_{0}\right] \geq y_{2} E_{0}\left[S_{b}\right]-y_{2} E\left[S_{0}\right]
$$

However, the consistency requirement rules out non-monotone equilibrium partitions for any belief function.

Proposition 7 All biased sorting equilibria with consistency satisfy monotonicity.

Proof. Suppose a non-monotone equilibrium exists. Then it must be the case that there exist $y_{1} \in S_{b}$ and $y_{2} \in S_{0}$ with $y_{1}<y_{2}$. Then the IC constraint for $y_{1}$ requires that

$$
y_{1} E_{b}\left[S_{0}\right] \leq y_{1} E\left[S_{b}\right]-b
$$

and note that this implies that $E\left[S_{b}\right]-E_{b}\left[S_{0}\right]>0$. The consistency requirement additionally requires that

$$
y_{2} E_{b}\left[S_{0}\right] \geq y_{2} E\left[S_{b}\right]-b .
$$

But these two conditions combined give

$$
y_{1} E\left[S_{b}\right]-y_{1} E_{b}\left[S_{0}\right] \geq y_{2} E\left[S_{b}\right]-y_{2} E_{b}\left[S_{0}\right],
$$

which cannot hold for any belief function $B$ if $y_{1}<y_{2}$, because as noted above $E\left[S_{b}\right]-E_{b}\left[S_{0}\right]>0$.

\subsection{Conditions for a unique equilibrium above the median}

Proposition 8 (Windsteiger (2017a)) If the belief function is such that the rich overestimate the poor group s average income, and the poor underestimate average income in the rich group, such that

$$
\underline{E}_{r}(\hat{y})>\underline{E}(\hat{y}) \quad \forall \hat{y} \in\left[0, y_{\max }\right)
$$

and

$$
\bar{E}(\hat{y})<\bar{E}_{p}(\hat{y}) \quad \forall \hat{y} \in\left(0, y_{\max }\right],
$$

and furthermore

$$
\underline{E}_{r}(\hat{y}) \in[0, \hat{y}] \text { and } \bar{E}_{p}(\hat{y}) \in\left[\hat{y}, y_{\max }\right]
$$


(which is a natural restriction on beliefs given that everybody knows where the cutoff $\hat{y}$ is) a binary biased sorting equilibrium with consistency always exists. If additionally the severity of the misperceptions is monotone in the cutoff, i.e.

$$
\frac{d\left(\bar{E}(\hat{y})-\bar{E}_{p}(\hat{y})\right)}{d \hat{y}}<0 \quad \text { and } \quad \frac{d\left(\underline{E}_{r}(\hat{y})-\underline{E}(\hat{y})\right)}{d \hat{y}}>0 \quad \forall \hat{y} \in\left(0, y_{\max }\right)
$$

the biased sorting equilibrium with consistency is unique.

Proof. Conditions (8), (9) and (10) together with the fact that $\underline{E}_{r}(\hat{y})$, $\underline{E}(\hat{y}), \bar{E}_{p}(\hat{y})$ and $\bar{E}(\hat{y})$ are continuous ensure existence. Condition (11) implies that people's misperceptions converge to the truth monotonically as $\hat{y}$ goes to 0 resp. $y_{\max }$ and hence there will be a unique $\hat{y}^{*}$ for which both groups have the same belief about the difference in average incomes (and thus about the benefits of sorting). For more explanations see Windsteiger (2017a).

Proposition 9 If both groups underestimate inequality, sufficient conditions for a unique equilibrium cutoff $\hat{y}^{*}$ above the median are conditions (8) to (11) and additionally

$$
\bar{E}_{p}\left(y^{M}\right)+\underline{E}_{r}\left(y^{M}\right)<2 E .
$$

Proof. Conditions (8) to (11) guarantee existence and uniqueness (see above). Concerning the last condition, note that if $\bar{E}-\bar{E}_{p}$ is monotonically increasing and $\underline{E}_{r}-\underline{E}$ is monotonically decreasing in $\hat{y}$, then

$$
\bar{E}_{p}(\hat{y})-\underline{E}(\hat{y})<\bar{E}(\hat{y})-\underline{E}_{r}(\hat{y})
$$

for all $\hat{y}$ below the unique equilibrium cutoff, and the inequality must hold in the other direction above the unique equilibrium cutoff. That implies

$$
\bar{E}_{p}(\hat{y})+\underline{E}_{r}(\hat{y})<\bar{E}(\hat{y})+\underline{E}(\hat{y})
$$

for all $\hat{y}$ below the equilibrium cutoff, and

$$
\bar{E}_{p}(\hat{y})+\underline{E}_{r}(\hat{y})>\bar{E}(\hat{y})+\underline{E}(\hat{y})
$$

for all $\hat{y}$ above the equilibrium cutoff. If the equilibrium should lie above the median, then at the median it must be the case that

$$
\bar{E}_{p}\left(y^{M}\right)+\underline{E}_{r}\left(y^{M}\right)<\bar{E}\left(y^{M}\right)+\underline{E}\left(y^{M}\right),
$$

because the median must be below the cutoff. The fact that

$$
E=\left(1-F\left(y^{M}\right)\right) \bar{E}\left(y^{M}\right)+F\left(y^{M}\right) \underline{E}\left(y^{M}\right)=\frac{\bar{E}\left(y^{M}\right)+\underline{E}\left(y^{M}\right)}{2}
$$


at the median proves the claim.

\subsection{Analysis of the unique binary biased sorting equi- librium}

As established in Section 3.1, any equilibrium cutoff is characterized by

$$
\hat{y}^{*}=\frac{a\left(1-F\left(\hat{y}^{*}\right)\right) \bar{E}\left(\hat{y}^{*}\right)+F\left(\hat{y}^{*}\right) \underline{E}\left(\hat{y}^{*}\right)}{a\left(1-F\left(\hat{y}^{*}\right)\right)+F\left(\hat{y}^{*}\right)}
$$

and hence it is the fixed point of

$$
h(\hat{y})=\frac{a(1-F(\hat{y})) \bar{E}(\hat{y})+F(\hat{y}) \underline{E}(\hat{y})}{a(1-F(\hat{y}))+F(\hat{y})} .
$$

Therefore, the equilibrium cutoff is exactly where the 45 degree line cuts the function $h$. As $\hat{y}^{*}$ approaches 0 , the left hand side of (12) becomes zero, while the right hand side becomes $h(0)=E$, and hence larger than the left hand side. As $\hat{y}^{*}$ approaches $y_{\max }$, the opposite happens: the left hand side becomes $y_{\max }$, and thus larger than the right hand side, which is again $h\left(y_{\max }\right)=E$. Hence, because the expressions on both sides are continuous in $\hat{y}$, we know that there must be a $\hat{y}$ in $\left(0, y_{\max }\right)$ for which equality holds. This concludes the proof that an equilibrium cutoff always exists in my model.

To ensure that there can only be one such intersection point, I can calculate

$$
h^{\prime}(\hat{y})=\frac{\left[\begin{array}{c}
\left(-a f(\hat{y}) \bar{E}(\hat{y})+a(1-F(\hat{y})) \frac{\partial \bar{E}(\hat{y})}{\partial \hat{y}}+f(\hat{y}) \underline{E}(\hat{y})+F(\hat{y}) \frac{\partial \underline{E}(\hat{y})}{\partial \hat{y}}\right)(a(1-F(\hat{y}))+F(\hat{y})) \\
-(a(1-F(\hat{y})) \bar{E}(\hat{y})+F(\hat{y}) \underline{E}(\hat{y}))(-a f(\hat{y})+f(\hat{y}))
\end{array}\right]}{(a(1-F(\hat{y}))+F(\hat{y}))^{2}}
$$

which can be simplified to

$$
h^{\prime}(\hat{y})=\frac{(1-a) f(\hat{y})}{(a(1-F(\hat{y}))+F(\hat{y}))^{2}}[a(1-F(\hat{y}))(\hat{y}-\bar{E}(\hat{y}))+F(\hat{y})(\hat{y}-\underline{E}(\hat{y}))] .
$$

This implies that $h$ has a local extremum or saddle point $\hat{y}^{* *}$ characterized by

$$
a\left(1-F\left(\hat{y}^{* *}\right)\right)\left(\hat{y}^{* *}-\bar{E}\left(\hat{y}^{* *}\right)\right)+F\left(\hat{y}^{* *}\right)\left(\hat{y}^{* *}-\underline{E}\left(\hat{y}^{* *}\right)\right)=0
$$

or equivalently

$$
\hat{y}^{* *}=\frac{a\left(1-F\left(\hat{y}^{* *}\right)\right) \bar{E}\left(\hat{y}^{* *}\right)+F\left(\hat{y}^{* *}\right) \underline{E}\left(\hat{y}^{* *}\right)}{a\left(1-F\left(\hat{y}^{* *}\right)\right)+F\left(\hat{y}^{* *}\right)}
$$


This is exactly the equation that characterizes the equilibrium cutoff and the fixed point of $h$, i.e. we find that $\hat{y}^{* *}=\hat{y}^{*}$. Whenever the 45 degree line cuts $h$ it must therefore be where the slope of $h$ is 0 . This means that at any intersection, the 45 degree line cuts $h$ from below, which implies that such an intersection can only happen once. It follows that $h$ will have a unique fixed point and the equilibrium cutoff is unique.

The fixed point of $h$ characterized by (13) (or equivalently (12)) is a local maximum if $a>1$ and a local minimum if $a<1$. This can be seen from noting that

$$
\begin{aligned}
h^{\prime \prime}(\hat{y})= & \frac{(1-a) f^{\prime}(\hat{y})}{(a(1-F(\hat{y}))+F(\hat{y}))^{2}}[a(1-F(\hat{y}))(\hat{y}-\bar{E}(\hat{y}))+F(\hat{y})(\hat{y}-\underline{E}(\hat{y}))] \\
& +\frac{(1-a) f(\hat{y})}{(a(1-F(\hat{y}))+F(\hat{y}))} \\
- & \frac{2(1-a)^{2} f^{2}(\hat{y})[a(1-F(\hat{y}))(\hat{y}-\bar{E}(\hat{y}))+F(\hat{y})(\hat{y}-\underline{E}(\hat{y}))]}{(a(1-F(\hat{y}))+F(\hat{y}))^{3}} .
\end{aligned}
$$

At $\hat{y}^{*}$ we know that

$$
a\left(1-F\left(\hat{y}^{*}\right)\right)\left(\hat{y}^{*}-\bar{E}\left(\hat{y}^{*}\right)\right)+F\left(\hat{y}^{*}\right)\left(\hat{y}^{*}-\underline{E}\left(\hat{y}^{*}\right)\right)=0
$$

and thus the first and the third term drop out of the second derivative and we get

$$
h^{\prime \prime}\left(\hat{y}^{*}\right)=\frac{(1-a) f\left(\hat{y}^{*}\right)}{\left(a\left(1-F\left(\hat{y}^{*}\right)\right)+F\left(\hat{y}^{*}\right)\right)} .
$$

As this expression is negative for $a$ larger than 1 and positive for $a$ smaller than $1, \hat{y}^{*}$ is a local maximum if $a>1$ and a local minimum at $a<1$. Figures 2 and 3 depict the intersection of $h$ and the 45 degree line for $a<1$ and $a>1$ (where the underlying income distribution is a truncated lognormal distribution). If $a=1$ the problem becomes very simple, as the expression for $h$ reduces to

$$
h(\hat{y})=E,
$$

i.e. $h$ is just a horizontal straight line at $E$ and the unique equilibrium cutoff is at $E$.

\subsection{The relationship between naivety and the equi- librium cutoff $\hat{y}^{*}$}

As noted in Section 3.1, the equilibrium cutoff depends on the naivety of the rich and the poor via a single parameter, $\frac{\beta}{\gamma}=a$, which describes the severity of the poor's naivety relative to the rich's. If $a=1$ then both groups are "equally naive", if $a>1$ then the poor are more naive than the 


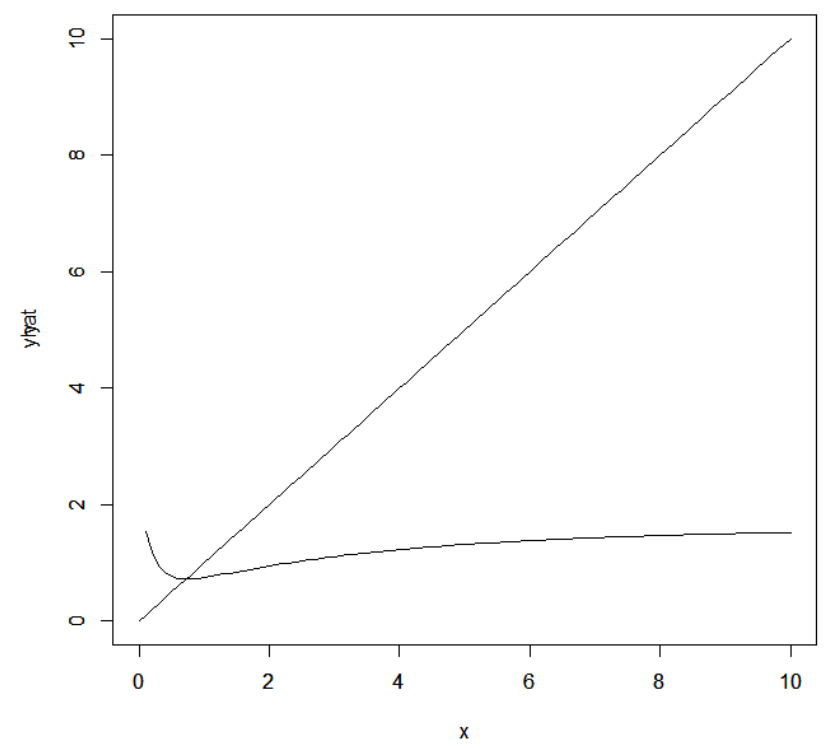

Figure 2: Equilibrium cutoff $\hat{y}^{*}$ if $a<1$

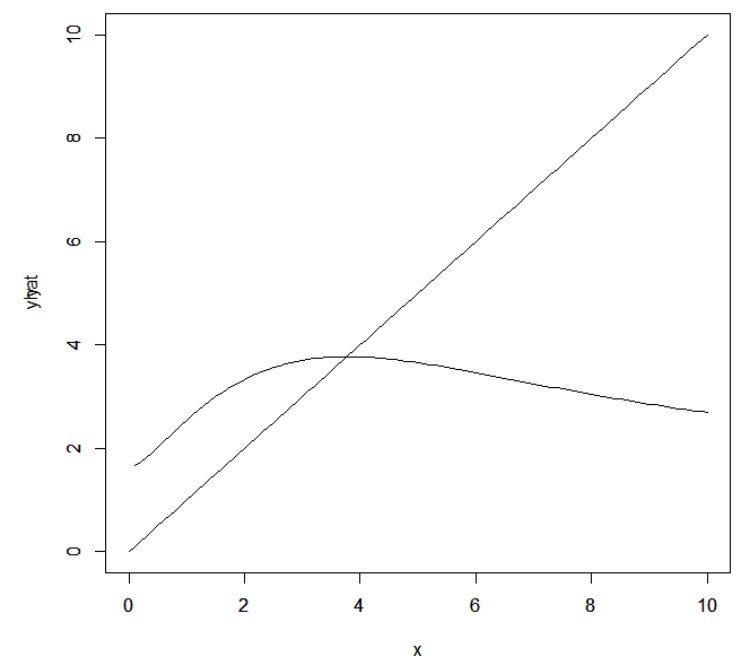

Figure 3: Equilibrium cutoff $\hat{y}^{*}$ if $a>1$ 
rich. Using the equilibrium condition

$$
\hat{y}^{*}=\frac{a\left(1-F\left(\hat{y}^{*}\right)\right) \bar{E}\left(\hat{y}^{*}\right)+F\left(\hat{y}^{*}\right) \underline{E}\left(\hat{y}^{*}\right)}{a\left(1-F\left(\hat{y}^{*}\right)\right)+F\left(\hat{y}^{*}\right)}
$$

I can investigate how $\hat{y}^{*}$ changes with $a$ :

$$
\begin{aligned}
& \left(1-F\left(\hat{y}^{*}\right)\right) \bar{E}\left(\hat{y}^{*}\right) d a+ \\
& \left(\begin{array}{c}
-a f\left(\hat{y}^{*}\right) \bar{E}\left(\hat{y}^{*}\right)+a\left(1-F\left(\hat{y}^{*}\right)\right) \frac{\bar{E}\left(\hat{y}^{*}\right)-\hat{y}^{*}}{1-F\left(\hat{y}^{*}\right)} f\left(\hat{y}^{*}\right)+f\left(\hat{y}^{*}\right) \underline{E}\left(\hat{y}^{*}\right) \\
+F\left(\hat{y}^{*}\right) \frac{\left(\hat{y}^{*}-\underline{E}\left(\hat{y}^{*}\right)\right)}{F\left(\hat{y}^{*}\right)} f\left(\hat{y}^{*}\right)
\end{array}\right) d \hat{y}^{*} \\
& =\left(a\left(1-F\left(\hat{y}^{*}\right)\right)+F\left(\hat{y}^{*}\right)+\hat{y}^{*}\left(-a f\left(\hat{y}^{*}\right)+f\left(\hat{y}^{*}\right)\right)\right) d \hat{y}^{*}+\left(1-F\left(\hat{y}^{*}\right)\right) \hat{y}^{*} d a \\
& \Longleftrightarrow(1-F)\left(\bar{E}-\hat{y}^{*}\right) d a=\left[a f \bar{E}-a\left(\bar{E}-\hat{y}^{*}\right) f-\underline{E} f-\left(\hat{y}^{*}-\underline{E}\right) f\right. \\
& \left.+a(1-F)+F+\hat{y}^{*} f(1-a)\right] d \hat{y}^{*} \\
& \Longleftrightarrow \frac{d \hat{y}^{*}}{d a}=\frac{\left(1-F\left(\hat{y}^{*}\right)\right)\left(\bar{E}\left(\hat{y}^{*}\right)-\hat{y}^{*}\right)}{a\left(1-F\left(\hat{y}^{*}\right)\right)+F\left(\hat{y}^{*}\right)}>0
\end{aligned}
$$

The equilibrium cutoff $\hat{y}^{*}$ is increasing in the degree of naivety of the poor relative to the rich. The higher $a$, the more the poor tend to underestimate the benefits of sorting (relative to the rich) and hence the more they need to see of the whole distribution relative to the rich to have the same perceived benefits of sorting as the rich.

As naivety goes to zero, what happens to the equilibrium cutoff depends on the speed of convergence of $\beta$ respectively $\gamma$. If $\beta$ converges to zero faster than $\gamma, a$ goes to zero and $\hat{y}^{*}$ goes to 0 . If $\gamma$ converges at a faster speed than $\beta$, $a$ converges to infinity and the equilibrium cutoff goes to $y_{\max }{ }^{22}$

\subsection{The consistency requirement}

The equilibrium condition in my model boils down to

$$
\hat{y}^{*}\left[\bar{E}\left(\hat{y}^{*}\right)-\underline{E}_{r}\left(\hat{y}^{*}\right)\right]=\hat{y}^{*}\left[\bar{E}_{p}\left(\hat{y}^{*}\right)-\underline{E}\left(\hat{y}^{*}\right)\right]
$$

and therefore the unique equilibrium cutoff $\hat{y}^{*}$ needs to satisfy

$$
\bar{E}\left(\hat{y}^{*}\right)-\underline{E}_{r}\left(\hat{y}^{*}\right)=\bar{E}_{p}\left(\hat{y}^{*}\right)-\underline{E}\left(\hat{y}^{*}\right)
$$

i.e. the perceived difference in group average incomes needs to be the same for both the rich and the poor group in equilibrium. Figure 4 depicts the poor group's (blue) and the rich group's (red) perceived group differences (in terms of average income) as well as the correct group differences (black)

\footnotetext{
${ }^{22}$ The best way to see the latter is to introduce the auxiliary parameter $b=\frac{\gamma}{\beta}$ in this case and rewrite $h(\hat{y})$ in terms of $b$.
} 


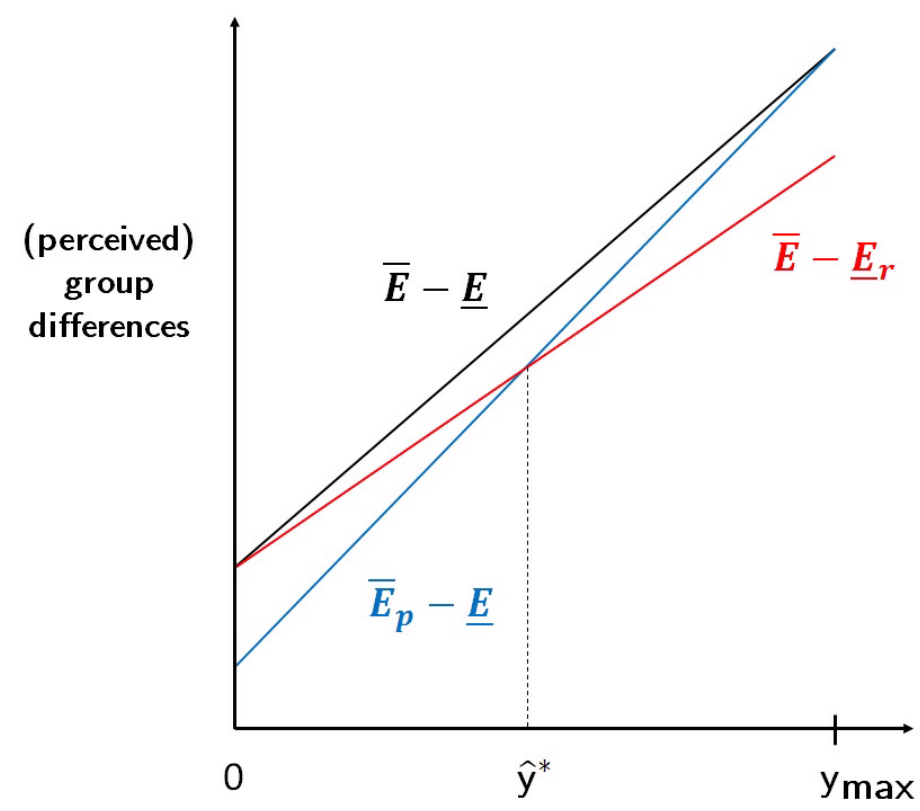

Figure 4: Perceived benefits of sorting for the rich (red) and poor (blue) and correct benefits of sorting as a function of the cutoff $\hat{y}$ (for a truncated lognormal distribution)

as a function of the cutoff $\hat{y}$ for the specific belief function used in this paper and defined by (2) and (3) (and with a truncated Lognormal as the underlying income distribution). For small $\hat{y}$, the rich perceive the difference between the two groups almost correctly, while the poor underestimate it a lot. This is due to the severity of misperceptions being monotone in group size: the larger the part of the income distribution that a group sees, the less biased they are about the other group. This also implies that as $\hat{y}$ increases, the rich become more and more biased and the poor become more and more correct about the group difference. The blue and the red line cross at $\hat{y}^{*}$, the unique binary biased sorting equilibrium with consistency, where both groups have the same assessment about the group differences. As $\hat{y}$ increases beyond this point, the poor group starts to value sorting more than the rich group.

What kind of equilibria would be possible if we do not require consistency? For a sorting equilibrium without consistency, the only condition that needs to be satisfied is that the cutoff is such that everybody in the rich group prefers being in the rich group to being in the poor group, while everybody in the poor group wants to stay in the poor group for some sorting fee $b>0$. In Figure 4 , all cutoffs $\hat{y}$ below $\hat{y}^{*}$ would satisfy this condition - if $\hat{y} \in\left(0, \hat{y}^{*}\right)$, the marginal person in the rich group values being in the rich group more than the marginal person in the poor group, and therefore we would be able to find a sorting fee $b>0$ that the rich are willing to pay, while it doesn't seem worthwhile for the poor to do so. 
Hence, all $\hat{y} \in\left(0, \hat{y}^{*}\right]$ are binary biased sorting equilibria. Meanwhile, none of the $\hat{y}$ above $\hat{y}^{*}$ can be biased sorting equilibria, because the marginal person in the poor group would always be willing to pay more to join the rich group than the marginal person in the rich group, and thus no $b>0$ could be found that separates the rich from the poor. Note however, that all $\hat{y} \in\left(0, \hat{y}^{*}\right)$, while constituting biased sorting equilibrium cutoffs, fail to satisfy the consistency requirement: Depending on the sorting fee (and note that the sorting fee is not unique if $\hat{y} \in\left(0, \hat{y}^{*}\right)$, any $b$ between $\hat{y}\left(\bar{E}_{p}-\underline{E}\right)$ and $\hat{y}\left(\bar{E}-\underline{E}_{r}\right)$ would work), either the people in the poor group would not understand why people at the bottom of the rich group want to pay $b$ to be part of the rich group (because for the poor, being in the rich group is worth less), or the rich would wonder why people at the top of the poor group don't want to join their group, or both happens at the same time (if $b$ is neither $\hat{y}\left(\bar{E}_{p}-\underline{E}\right)$ nor $\hat{y}\left(\bar{E}-\underline{E}_{r}\right)$ but somewhere in between).

In the specific case analyzed here, the consistency requirement selects a unique equilibrium out of the range of sorting equilibria. This is because the misperceptions converge to the truth monotonically, which implies that the blue line approaches the black line monotonically as the cutoff increases, while the red line approaches the black line monotonically as $\hat{y}$ decreases. Therefore, the two lines can only cut once. If the misperceptions were not monotone, the distance between the black line and the blue resp. red line could be non-monotone, and therefore the blue and the red line could intersect several times. Each of those intersections would then constitute a biased sorting equilibrium with consistency. Consistency alone is not enough to guarantee uniqueness. Consistency and monotonicity of the misperceptions together do the job.

To summarize the above: without consistency, there is in general no clear prediction of how groups will look like in equilibrium, because uniqueness of equilibrium is not guaranteed. With the specific belief function used in the paper, any cutoff $\hat{y} \in\left(0, \hat{y}^{*}\right]$ can be supported as a sorting equilibrium. Would the main results of my paper still hold if we abandon the consistency requirement? Leaving asside the fact that doing comparative statics is tricky in the absence of a unique equilibrium (because the model yields no prediction of how the group partition will be affected as inequality changes), the central prediction of the model - namely that perceived inequality (and hence demand for redistribution) can go down if inequality increases - continues to hold as long as the median earner is in the poor group before and after the change in the income distribution. Note though that people's misperceptions depend on their group's relative size, and hence in order to predict how perceived inequality changes due to increases in inequality we would need a theory of how the groups change due to changes in the income distribution, which we lack if the equilibrium partition is not unique. 
Before I close this section, I would like to give another justification for why refining equilibria to satisfy consistency makes sense: We can also interpret the consistency requirement is a refinement to "no-learning partitions". If a partition satisfies consistency, then people never come across anything that goes against their beliefs and surprises them, therefore they have no impulse to modify their beliefs or their actions in any way.

I do not model any form of learning in this paper. I also do not make any assumptions about what happens if people encounter other people, whose choices they do not understand. One possibility is that people just assume that the others are wrong if they are puzzled by their choices, and do not modify their own beliefs or actions. Another possibility is that they start to question their own beliefs about the other groups and maybe try to update them, based on choices of other people that they observe. Alternatively, they might even experiment and join another group to learn about average income in that group. The consistency requirement restricts the set of biased sorting equilibria to those partitions where neither of the above happens, because people are simply not puzzled by anybody else's choices. In that sense, the consistency requirement can be viewed as a stability refinement: consistent equilibrium partitions are stable with respect to learning, experimenting or updating. Because what they see is consistent with their beliefs about the world, people have no incentives to question or change their beliefs, and thus the partition is stable irrespective of what they would do if they would encounter anything that is at odds with their beliefs.

\subsection{A median-preserving spread of the lognormal dis- tribution and monopolist profits}

Recall that the monopolist's profits from offering one cutoff (which in equilibrium will be at $E$ if $a=1$ ) can be written as

$$
E(E-\underline{E})[1-\gamma F(E)(1-F(E))]-c
$$

For the lognormal distribution, this becomes

$\Pi=E\left[E\left(1-\frac{\Phi\left(\frac{\ln (E)-\mu}{\sigma}-\sigma\right)}{\Phi\left(\frac{\ln (E)-\mu}{\sigma}\right)}\right)\left(1-\gamma \Phi\left(\frac{\ln (E)-\mu}{\sigma}\right)+\gamma\left[\Phi\left(\frac{\ln (E)-\mu}{\sigma}\right)\right]^{2}\right)\right]-c$

which can be simplified to

$$
\Pi=E\left[E\left(1-\frac{1-\Phi\left(\frac{\sigma}{2}\right)}{\Phi\left(\frac{\sigma}{2}\right)}\right)\left(1-\gamma \Phi\left(\frac{\sigma}{2}\right)+\gamma\left[\Phi\left(\frac{\sigma}{2}\right)\right]^{2}\right)\right]-c
$$




$$
=E^{2}\left(\frac{2 \Phi\left(\frac{\sigma}{2}\right)-1}{\Phi\left(\frac{\sigma}{2}\right)}\right)\left(1-\gamma \Phi\left(\frac{\sigma}{2}\right)+\gamma\left[\Phi\left(\frac{\sigma}{2}\right)\right]^{2}\right)-c
$$

because $\ln \hat{y}=\mu+\sigma^{2}$ if $\hat{y}=E$.

I find that

$$
\begin{aligned}
\frac{d \Pi}{d \sigma}= & 2 \sigma E^{2}\left(\frac{2 \Phi\left(\frac{\sigma}{2}\right)-1}{\Phi\left(\frac{\sigma}{2}\right)}\right)\left(1-\gamma \Phi\left(\frac{\sigma}{2}\right)+\gamma\left[\Phi\left(\frac{\sigma}{2}\right)\right]^{2}\right) \\
& +E^{2}\left[\frac{\phi\left(\frac{\sigma}{2}\right) \frac{1}{2}}{\Phi\left(\frac{\sigma}{2}\right)}\right]\left(1-\gamma \Phi\left(\frac{\sigma}{2}\right)+\gamma\left[\Phi\left(\frac{\sigma}{2}\right)\right]^{2}\right) \\
& +E^{2}\left(\frac{2 \Phi\left(\frac{\sigma}{2}\right)-1}{\Phi\left(\frac{\sigma}{2}\right)}\right) \gamma \phi\left(\frac{\sigma}{2}\right)\left(\Phi\left(\frac{\sigma}{2}\right)-\frac{1}{2}\right)
\end{aligned}
$$

As $\Phi\left(\frac{\sigma}{2}\right)>\frac{1}{2}$, all of the terms are positive and hence the monopolist's profit always increases if $\sigma$ increases.

Proposition 10 If income is lognormally distributed, an increase in inequality in the form of a median-preserving spread increases the monopolist's revenues from offering the sorting technology.

\subsection{Sufficient conditions for Assumption 1}

$$
\begin{gathered}
\frac{y^{M}}{E_{p}} \leq \frac{E}{E_{r}} \\
\Longleftrightarrow y^{M}\left(F \underline{E}_{r}+(1-F) \bar{E}\right) \leq E\left(F \underline{E}+(1-F) \bar{E}_{p}\right)
\end{gathered}
$$

If $\beta=\gamma$, this can be simplified to

$$
\beta\left(y^{M} F^{2}(E-\underline{E})+E(1-F)^{2}(\bar{E}-E)\right) \leq E\left(E-y^{M}\right)
$$

Noting that

$$
E-\underline{E}=(1-F)(\bar{E}-\underline{E})
$$

and

$$
\bar{E}-E=F(\bar{E}-\underline{E})
$$

I can further simplify to

$$
\beta F(1-F)\left(F \frac{y^{M}}{E}+(1-F)\right)(\bar{E}-\underline{E}) \leq\left(E-y^{M}\right)
$$


Given that $F(1-F)<0.25$ (because $y^{M}<E$ ) and $\frac{y^{M}}{E}<1$, I have that

$$
\beta F(1-F)\left(F \frac{y^{M}}{E}+(1-F)\right)(\bar{E}-\underline{E})<\beta \frac{(\bar{E}-\underline{E})}{4}
$$

and it follows that

$$
\beta \frac{(\bar{E}-\underline{E})}{4} \leq E-y^{M}
$$

is a sufficient condition for

$$
\frac{y^{M}}{E_{p}} \leq \frac{E}{E_{r}}
$$

(in fact it is even a sufficient condition for $\frac{y^{M}}{E_{p}}<\frac{E}{E_{r}}$, given that inequality (16) is strict). This condition holds if $E-y^{M}$ is large enough compared to $\bar{E}(E)-\underline{E}(E)$, i.e. if the distribution is positively skewed but there is not too much mass at the tails of the distribution, and if $\beta$ is small, i.e. people are not too biased.

\subsection{Detailed calculations for Section 4.2}

Average income $E$ does not change due to a mean-preserving spread and hence $^{23}$

$$
\Delta E=F \Delta \underline{E}+(1-F) \Delta \bar{E}=0,
$$

Average perceived income of the poor, $E_{p}$, declines, because

$$
\Delta E_{p}=F \Delta \underline{E}+(1-F) \Delta \bar{E}_{p}
$$

and

$$
\bar{E}_{p}(\hat{y})=\beta(1-F) \hat{y}+(1-\beta(1-F)) \bar{E}
$$

which implies

$$
\Delta \bar{E}_{p}(E)=(1-\beta(1-F)) \Delta \bar{E}<\Delta \bar{E}
$$

(as $\hat{y}=E$ doesn't change). The change in $\frac{y^{M}}{E_{p}}$ amounts to

$$
\Delta\left(\frac{y^{M}}{E_{p}}\right)=\frac{\Delta y^{M} E_{p}-y^{M} \Delta E_{p}}{\left(E_{p}\right)^{2}}=\left(\frac{\Delta y^{M}}{y^{M}}-\frac{\Delta E_{p}}{E_{p}}\right) \frac{y^{M}}{E_{p}}
$$

and thus the percentage change in $\frac{y^{M}}{E_{p}}$ is $\frac{\Delta y^{M}}{y^{M}}-\frac{\Delta E_{p}}{E_{p}}$, which is smaller (in absolute terms) than the percentage change of $\frac{y^{M}}{E}$ in the unbiased case, because $\frac{\Delta E_{p}}{E_{p}}<0$. In the following I show that if $\left|\frac{\Delta E_{p}}{E_{p}}\right|$ is large enough

\footnotetext{
${ }^{23}$ And note that I require the mean-preserving spread to be such that $F\left(\hat{y}^{*}\right)=F(E)$ doesn't change.
} 
relative to $\left|\frac{\Delta y^{M}}{y^{M}}\right|$, the median earner will even think that inequality has decreased, i.e. the percentage change in $\frac{y^{M}}{E_{p}}$ (and hence also the absolute change in $\left.\frac{y^{M}}{E_{p}}\right)$ can be positive:

From (17) and (18) it follows that

$$
\Delta E_{p}(E)=-(1-F) \Delta \bar{E}+(1-F) \Delta \bar{E}_{p}(E)=-\beta(1-F)^{2} \Delta \bar{E}(E)
$$

Furthermore,

$$
E_{p}(E)=F \underline{E}(E)+(1-F) \bar{E}_{p}(E)=E-\beta(1-F)^{2}(\bar{E}(E)-E)
$$

and therefore

$$
\frac{\Delta E_{p}}{E_{p}}=\frac{-\beta(1-F)^{2} \Delta \bar{E}}{E-\beta(1-F)^{2}(\bar{E}-E)}=\frac{\beta(1-F) F \Delta \underline{E}}{E-\beta(1-F)^{2}(\bar{E}-E)}
$$

(using (17) again). Hence, I get

$$
\begin{gathered}
\frac{\Delta y^{M}}{y^{M}}-\frac{\Delta E_{p}}{E_{p}}>0 \Longleftrightarrow \frac{\Delta y^{M}}{y^{M}}>\frac{\beta(1-F) F \Delta \underline{E}}{E-\beta(1-F)^{2}(\bar{E}-E)} \\
\Longleftrightarrow \frac{\Delta y^{M}}{\Delta \underline{E}}<\frac{\beta y^{M}(1-F) F}{E-\beta(1-F)^{2}(\bar{E}-E)}
\end{gathered}
$$

(where both sides are positive). For a given $\frac{\Delta y^{M}}{\Delta \underline{E}}$ this condition is more likely to be satisfied if $\beta$ is large, because

$$
\frac{\partial}{\partial \beta}\left(\frac{\beta}{E-\beta(1-F)^{2}(\bar{E}-E)}\right)=\frac{E}{\left[E-\beta(1-F)^{2}(\bar{E}-E)\right]^{2}}>0
$$

and hence the RHS is increasing in $\beta$. Furthermore, $(1-F(E)) F(E)$ should not be too small, i.e. the income distribution cannot be too positively skewed, such that $F(E)$ is not too far above 0.5. Note however, that such a monotone mean-preserving spread can be constructed for any given income distribution, by ensuring that $\Delta y^{M}$ and $\Delta \underline{E}$ are such that (19) holds. To see this, note that we need to ensure that Assumption 1 is satisfied before and after the mean-preserving spread. A sufficient condition for this is that

$$
\beta \frac{(\bar{E}(E)-\underline{E}(E))}{4} \leq E-y^{M}
$$

holds (see Appendix 7.7). If the mean-preserving spread would be such that $\Delta y^{M}=0$ (i.e. all the mass shifts around below and above the median, but the median stays the same), then (19) would be satisfied. If this mean-preserving spread is such that $\Delta \underline{E}$ (and corresponding $\Delta \bar{E}$ ) are small 
enough (in absolute value) and therefore Assumption 1 still holds after the change $^{24}$, this mean-preserving spread would lead to a decrease in demand for redistribution (whereas in the unbiased case demand for redistribution would not change because neither $y^{M}$ nor $E$ has changed). Because all the expressions are continuous, it follows that we can analogously construct a mean-preserving spread that satisfies (20) and has $\Delta y^{M}>0$ (and where thus demand for redistribution increases in the unbiased case, but decreases in the presence of misperceptions). Hence, I can conclude that

Lemma 3 For any $\beta>0$ there exists a mean-preserving spread of the income distribution such that an increase in inequality leads to a decrease in the median earner's perceived degree of inequality.

Now let me examine the absolute change of $\frac{y^{M}}{E}$ and $\frac{y^{M}}{E_{p}}$ : I want to derive sufficient conditions for the absolute decrease in perceived equality to be smaller under segregation, i.e.

$$
\Delta\left(\frac{y^{M}}{E}\right)<\Delta\left(\frac{y^{M}}{E_{p}}\right)
$$

(because both sides of this inequality are negative). Lemma 3 shows that I can always construct a mean-preserving spread satisfying (19) such that perceived equality $\frac{y^{M}}{E_{p}}$ increases under segregation (in which case inequality (21) trivially holds, because $\frac{y^{M}}{E}$ will always decrease). However, less strong conditions can be derived in order for (21) to hold without perceived inequality having to decrease:

$$
\begin{gathered}
\Delta\left(\frac{y^{M}}{E}\right)<\Delta\left(\frac{y^{M}}{E_{p}}\right) \Longleftrightarrow \\
\frac{\Delta y^{M}}{E}<\frac{\Delta y^{M} E_{p}-y^{M} \Delta E_{p}}{\left(E_{p}\right)^{2}}=\frac{\Delta y^{M}}{E_{p}}-\frac{y^{M} \Delta E_{p}}{\left(E_{p}\right)^{2}} \\
\Longleftrightarrow \frac{\Delta y^{M}}{E}<\frac{\Delta y^{M}}{E-\beta(1-F)^{2}(\bar{E}-E)}+\frac{y^{M} \beta(1-F)^{2} \Delta \bar{E}}{\left(E-\beta(1-F)^{2}(\bar{E}-E)\right)^{2}} \\
\Longleftrightarrow \Delta y^{M}\left(\frac{1}{E}-\frac{1}{E-\beta(1-F)^{2}(\bar{E}-E)}\right)<\frac{y^{M} \beta(1-F)^{2} \Delta \bar{E}}{\left(E-\beta(1-F)^{2}(\bar{E}-E)\right)^{2}} \\
\Longleftrightarrow \frac{\Delta y^{M}}{y^{M}}\left(\frac{-\beta(1-F)^{2}(\bar{E}-E)}{E\left(E-\beta(1-F)^{2}(\bar{E}-E)\right)}\right)<\frac{\beta(1-F)^{2} \Delta \bar{E}}{\left(E-\beta(1-F)^{2}(\bar{E}-E)\right)^{2}}
\end{gathered}
$$

\footnotetext{
${ }^{24} \mathrm{By}$ continuity, such a mean-preserving spread can always be found because if (20) is satisfied initially then $\frac{y^{M}}{E_{p}}$ is strictly smaller than $\frac{E}{E_{r}}$ (see Appendix 7.7) and hence a small change in $\bar{E}$ and $\underline{E}$ will still leave $\frac{y^{M}}{E_{p}} \leq \frac{E}{E_{r}}$.
} 


$$
\begin{gathered}
\Longleftrightarrow \frac{\Delta y^{M}}{y^{M}}\left(\frac{-(\bar{E}-E)}{E}\right)<\frac{\Delta \bar{E}}{E-\beta(1-F)^{2}(\bar{E}-E)} \\
\Longleftrightarrow \frac{\Delta y^{M}}{y^{M}}\left(\frac{-F(\bar{E}-\underline{E})}{E}\right)<\frac{\frac{-F \Delta \underline{E}}{1-F}}{E-\beta(1-F)^{2}(\bar{E}-E)} \\
\Longleftrightarrow-\frac{\Delta y^{M}}{y^{M}}\left(\frac{(1-F)(\bar{E}-\underline{E})}{E}\right)<\frac{-\Delta \underline{E}}{E-\beta(1-F)^{2}(\bar{E}-E)} \\
\Longleftrightarrow \frac{\Delta y^{M}}{\Delta \underline{E}}\left(\frac{E-\underline{E}}{E}\right)<\frac{y^{M}}{E-\beta(1-F)^{2}(\bar{E}-E)} \\
\Longleftrightarrow \frac{\Delta y^{M}}{\Delta \underline{E}}<\frac{y^{M} E}{\left(E-\beta(1-F)^{2}(\bar{E}-E)\right)(E-\underline{E})}=\frac{y^{M} E}{E_{p}(E-\underline{E})}
\end{gathered}
$$

For a given mean-preserving spread, this inequality is more likely to hold if $\beta$ is large (such that $E_{p}$ is small relative to $E$ ). Note however, that it is always possible to construct a mean-preserving spread that satisfies this inequality, by designing $\Delta y^{M}$ and $\Delta \underline{E}$ accordingly.

Lemma 4 The (absolute) decrease in $\frac{y^{M}}{E_{p}}$ is smaller than the (absolute) decrease in $\frac{y^{M}}{E}$ iff the mean-preserving spread is such that

$$
\frac{\Delta y^{M}}{\Delta \underline{E}}<\frac{y^{M} E}{\left(E-\beta(1-F)^{2}(\bar{E}-E)\right)(E-\underline{E})} .
$$

In the absence of segregation, the change in the median earner's preferred tax rate due to a mean-preserving spread is given by ${ }^{25}$

$$
\Delta t^{*}=\tau^{\prime-1}\left(\frac{\dot{y}^{M}}{E}\right)-\tau^{\prime-1}\left(\frac{y^{M}}{E}\right) .
$$

If society is segregated, the change in the median earner's preferred tax rate amounts to ${ }^{26}$

$$
\Delta \tilde{t}^{*}=\tau^{\prime-1}\left(\frac{\dot{y}^{M}}{\dot{E}_{p}}\right)-\tau^{\prime-1}\left(\frac{y^{M}}{E_{p}}\right) .
$$

If the conditions of Lemma (4) hold, the decrease in $\frac{y^{M}}{E_{p}}$ is smaller than the decrease in $\frac{y^{M}}{E}$. Furthermore, I know that $\frac{y^{M}}{E_{p}}>\frac{y^{M}}{E}$. Together with the fact that $\tau^{\prime \prime}(t) \leq 0$ and $\tau^{\prime \prime \prime}(t) \geq 0$, which implies that $\tau^{-1}$ is decreasing and convex, this gives

$$
\Delta \tilde{t}^{*}<\Delta t^{*}
$$

\footnotetext{
${ }^{25}$ Notation: $\dot{y}^{M}$ denotes median income after the mean-preserving spread.

${ }^{26}$ Notation: $\dot{E}_{p}$ denotes the poor group's perception of average income after the meanpreserving spread.
} 
Lemma 5 If the mean-preserving spread is such that (22) holds, the increase in the preferred tax rate is less in a segregated society than in the absence of segregation.

\subsection{Detailed calculations for Section 4.3}

If a mean-preserving spread leads to economic segregation, the median earner's demand for redistribution declines if

$$
\begin{gathered}
\frac{y^{M}}{E}<\frac{\dot{y}^{M}}{E_{p}} \\
\Longleftrightarrow y^{M}\left(E-\beta(1-F)^{2}(\bar{E}+\Delta \bar{E}-E)\right)<y^{M} E+E \Delta y^{M} \\
\Longleftrightarrow \frac{\beta(1-F)^{2}(\bar{E}+\Delta \bar{E}-E)}{E}>-\frac{\Delta y^{M}}{y^{M}}=\left|\frac{\Delta y^{M}}{y^{M}}\right|
\end{gathered}
$$

For a given mean-preserving spread this inequality holds if $\beta$ is large enough (i.e. people are sufficiently naive) and the increase in average income in the rich group is large enough relative to the decline in median income. Again, it is immediate to see that a mean-preserving spread satisfying (23) can always be constructed by designing $\Delta \bar{E}$ and $\Delta y^{M}$ accordingly (the proof is analogous to the proof of Lemma 3 in Appendix 7.8).

\subsection{The effect of general changes in the shape of the income distribution on the demand for redistri- bution if society is segregated}

What happens to people's preferred redistribution rate if inequality between groups changes when people are already segregated? First and foremost this depends on how this change affects the equilibrium cutoff $\hat{y}^{*}$. Recall that the equilibrium cutoff is given by

$$
\hat{y}^{*}=\frac{a\left(1-F\left(\hat{y}^{*}\right)\right) \bar{E}\left(\hat{y}^{*}\right)+F\left(\hat{y}^{*}\right) \underline{E}\left(\hat{y}^{*}\right)}{a\left(1-F\left(\hat{y}^{*}\right)\right)+F\left(\hat{y}^{*}\right)},
$$

i.e. $\hat{y}^{*}$ is the fixed point of the function

$$
h(\hat{y})=\frac{a(1-F(\hat{y})) \bar{E}(\hat{y})+F(\hat{y}) \underline{E}(\hat{y})}{a(1-F(\hat{y}))+F(\hat{y})} .
$$

As described in Section 3.1 and Appendix 7.3, (24) has a unique fixed point. If $a=1$, this fixed point is at average income $E$. For $a<1$ the intersection between $h(\hat{y})$ and the 45 degree line looks like Figure 2, if $a>1$ then it looks like Figure 3 (and if $a=1, \hat{y}^{*}$ is where the 45 degree line intersects 
with the horizontal line at $E$ ). From these graphs it is immediate to see that the impact of an increase in inequality on the equilibrium cutoff depends on how this increase in inequality affects $h(\hat{y})$ (and thus the intersection of the 45 degree line with $h(\hat{y}))$.

If $\underline{E}\left(\hat{y}^{*}\right)$ goes down while $F\left(\hat{y}^{*}\right)$ and $\bar{E}\left(\hat{y}^{*}\right)$ stay the same, $h(\hat{y})$ shifts down, and the intersection with the 45 degree line (= the equilibrium cutoff $\hat{y}^{*}$ ) goes down (both if $a>1$ and if $a<1$ ). Hence, the new equilibrium cutoff will be lower. The opposite happens if $\bar{E}$ goes up ceteris paribus, i.e. if the rich group gets richer on average: Then it is straightforward to see from (24) that the new equilibrium cutoff will be higher.

Suppose that both things happen, so $\bar{E}$ increases, while $\underline{E}$ decreases (while $F\left(\hat{y}^{*}\right)$ doesn't change). Then whether the new equilibrium cutoff is higher or lower than the old one depends on $a$ and $F\left(\hat{y}^{*}\right)\left(\right.$ resp. $1-F\left(\hat{y}^{*}\right)$ ): if $a$ is high, or $1-F\left(\hat{y}^{*}\right)$ is high, such that $a(1-F) \Delta \bar{E}+F \Delta \underline{E}>0$, then the new equilibrium cutoff will be higher, if $a$ and/or $1-F$ is low, then the new equilibrium cutoff will be lower. If $a=1$ (meaning both groups are equally naive) then the cutoff is always $E$ and hence will go down if $E$ decreases due to this increase in inequality. $E$ decreases if $F(E)$ is high and $(1-F(E))$ is low, a feature that characterizes unequal distributions with positive skew.

If $\underline{E}$ decreases by $\Delta \underline{E}$ ceteris paribus, then as I have argued above, $\hat{y}^{*}$ will go down. What happens to preferences for redistribution depends on the position of $y^{M}$ : If $a=1$, the equilibrium cutoff is always at $E$, hence $y^{M}<\hat{y}^{*}=E$ before and after the decline in $\underline{E}$. If $y^{M}$ is sufficiently below the cutoff, such that preferences for redistribution do not overlap (i.e. if Assumption 1 is satisfied at all times) the median earner is the decisive voter both before and after the change in $\underline{E}$. Under these circumstances, a decrease in $\underline{E}$ and subsequently in $\hat{y}^{*}$ will mean that $\underline{E}$ decreases by $\Delta \underline{E}+\frac{\partial \underline{E}}{\partial \hat{y}} d \hat{y}^{*}, \bar{E}$ decreases by $\frac{\partial \bar{E}}{\partial \hat{y}} d \hat{y}^{*}$ and $E$ decreases by $F \Delta \underline{E}$ (the decreases in $\underline{E}$ and $\bar{E}$ due to the decrease in $\hat{y}^{*}$ cancel out with changes in $F$ and $1-F$ and do not affect $E$ : clearly, where the cutoff is has no implications for average income). The decrease in $\underline{E}$ and subsequent fall in $\hat{y}^{*}$ will lead to a decrease in $\bar{E}_{p}$ for two reasons: because $\bar{E}$ decreases due to the decline in $\hat{y}^{*}$ and because as $\hat{y}^{*}$ decreases, the poor become more biased, i.e. $\bar{E}-\bar{E}_{p}$ increases. As $\bar{E}_{p}$ decreases, clearly also $E_{p}$ decreases, and if the poor are sufficiently biased, then this can lead to a situation where the perceived equality ratio $\frac{y^{M}}{E_{p}}$ does not decrease (as the true equality ratio unambiguously will), but instead increases, because $E_{p}$ decreases by more than $y^{M}$ :

$$
d\left(\frac{y^{M}}{E_{p}}\right)=\frac{\Delta y^{M} E_{p}-y^{M} d E_{p}}{\left(E_{p}\right)^{2}}=\left(\frac{\Delta y^{M}}{y^{M}}-\frac{d E_{p}}{E_{p}}\right) \frac{y^{M}}{E_{p}}
$$


It can be shown that

$$
d E_{p}=F \Delta \underline{E}+(1-F) \beta[(1-F)+f(\bar{E}-\hat{y})] d \hat{y}^{*}
$$

and hence (note that both $\Delta \underline{E}$ and $d \hat{y}^{*}$ are negative)

$$
\left|d E_{p}\right|>|F \Delta \underline{E}|=|d E| \text {. }
$$

This implies that

$$
\left|\frac{d E_{p}}{E_{p}}\right|>\left|\frac{d E}{E}\right|
$$

(because we also have that $E_{p}<E$ ) and therefore the percentage decline in the perceived equality ratio, $\frac{\Delta y^{M}}{y^{M}}-\frac{d E_{p}}{E_{p}}$, will always be smaller than the percentage decline in the true equality ratio, $\frac{\Delta y^{M}}{y^{M}}-\frac{d E}{E}$. Moreover, the decline in $\underline{E}$ can be such that

$$
\frac{\Delta y^{M}}{y^{M}}-\frac{d E}{E}<0
$$

while

$$
\frac{\Delta y^{M}}{y^{M}}-\frac{d E_{p}}{E_{p}}>0
$$

because $E_{p}$ decreases by more than $y^{M}$. In fact it can be calculated that this will be the case if the decline in $\underline{E}$ is such that

$$
\frac{\Delta y^{M}}{F \Delta \underline{E}+(1-F) \beta[(1-F)+f(\bar{E}-\hat{y})] d \hat{y}^{*}}<\frac{y^{M}}{E_{p}} .
$$

Note that if $\underline{E}$ decreases ceteris paribus we have that

$$
d \hat{y}^{*}=\frac{F \Delta \underline{E}}{a(1-F)+F}
$$

This can be deduced from taking the total derivative of (24) which yields

$$
d \hat{y}^{*}=h^{\prime}\left(\hat{y}^{*}\right) d \hat{y}^{*}+\frac{F \Delta \underline{E}}{a(1-F)+F}
$$

and noting that $h^{\prime}\left(\hat{y}^{*}\right)=0$ (see Appendix 7.3). Hence, if the decline in $\underline{E}$ is such that

$$
\frac{\Delta y^{M}}{F \Delta \underline{E}\left(1+\frac{(1-F) \beta[(1-F)+f(\bar{E}-\hat{y})]}{a(1-F)+F}\right)}<\frac{y^{M}}{E_{p}}
$$

the true equality ratio $\frac{y^{M}}{E}$ decreases, while the perceived equality ratio $\frac{y^{M}}{E_{p}}$ 
increases - a change in inequality that leads to a decrease of $\frac{y^{M}}{E}$ if people are unbiased, will lead to an increase in $\frac{y^{M}}{E_{p}}$ in the biased case. Therefore, if $\underline{E}$ decreases, the new preferred tax rate after this increase in inequality can be lower than before. An increase in inequality can lead to a decrease in the demand for redistribution due to people's biased perception of the average income change in the other group and the change in group composition, which affects people's bias.

Suppose that instead of $\underline{E}$ decreasing, $\bar{E}$ increases by $\Delta \bar{E}$. Then the above analysis yields that $\hat{y}^{*}$ must increase - an initial increase in $\bar{E}$ by $\Delta \bar{E}$ means that the new equilibrium cutoff of the biased sorting equilibrium has to be higher. This implies that the total increase in $\bar{E}$ will be the sum of the shift $\Delta \bar{E}$ and the effect on $\bar{E}$ due to an increase in $\hat{y}^{*}$ :

$$
d \bar{E}=f \frac{(\bar{E}-\hat{y})}{(1-F)} d \hat{y}^{*}+\Delta \bar{E}
$$

Furthermore, also $\underline{E}$ increases due to the change in the cutoff. Hence, I have

$$
d E_{p}=\left[f \underline{E}+F \frac{\partial \underline{E}}{\partial \hat{y}}\right] d \hat{y}^{*}+(1-F) d \bar{E}_{p}-f \bar{E}_{p} d \hat{y}^{*}
$$

Remember that $\bar{E}_{p}=E-\beta(1-F)\left(\bar{E}-\hat{y}^{*}\right)$ and therefore

$$
d \bar{E}_{p}=d \bar{E}-\beta(1-F) d \bar{E}+\beta(1-F)\left(\bar{E}-\hat{y}^{*}\right) d \hat{y}^{*}
$$

Using (25) and (26), I get

$$
d E_{p}=(1-F) \Delta \bar{E}-\beta(1-F)^{2} \Delta \bar{E}+(1-F) \beta[(1-F)+f(\bar{E}-\hat{y})] d \hat{y}^{*}
$$

and hence $E_{p}$ increases in this case (both $\Delta \bar{E}$ and $d \hat{y}^{*}$ are positive here). Because $\hat{y}^{*}$ increases, the poor group is getting larger and therefore less biased, which means $E_{p}$ gets closer to $E$ and thus increases for two reasons: because $E$ increases, and because the poor become less biased and underestimate average income by less.

The fact that both $E$ and $E_{p}$ increase implies that the perceived equality ratio $\frac{y^{M}}{E_{p}}$ will move in the same direction as the true equality ratio $\frac{y^{M}}{E}$, namely it will decrease due to an increase in the denominator (note that the numerator $y^{M}$ doesn't change in this case because only the part of the income distribution that lies above $\hat{y}^{*}$ changes if $\bar{E}$ increases ceteris paribus). Under certain conditions, the percentage decrease in perceived equality can even be larger than the percentage decrease in true equality. 
The percentage decrease in perceived equality amounts to

$$
\frac{d\left(\frac{y^{M}}{E_{p}}\right)}{\frac{y^{M}}{E_{p}}}=-\frac{d E_{p}}{E_{p}}
$$

whereas the percentage decrease in true equality is

$$
\frac{d\left(\frac{y^{M}}{E}\right)}{\frac{y^{M}}{E}}=-\frac{\Delta E}{E}
$$

In order for the percentage decrease in perceived inequality to be higher (in absolute value) we need

$$
\frac{d E_{p}}{E_{p}}>\frac{\Delta E}{E}
$$

which can be rewritten as

$$
\frac{(1-F) \Delta \bar{E}-\beta(1-F)^{2} \Delta \bar{E}+(1-F) \beta[(1-F)+f(\bar{E}-\hat{y})] d \hat{y}^{*}}{E-\beta(1-F)^{2}\left(\bar{E}-\hat{y}^{*}\right)}>\frac{(1-F) \Delta \bar{E}}{E}
$$

Using

$$
d \hat{y}^{*}=h^{\prime}\left(\hat{y}^{*}\right) d \hat{y}^{*}+\frac{a(1-F) \Delta \bar{E}}{a(1-F)+F}=\frac{a(1-F) \Delta \bar{E}}{a(1-F)+F}
$$

(because $h^{\prime}\left(\hat{y}^{*}\right)=0$ ) this becomes, after simplifying,

$$
\frac{f\left(\bar{E}-\hat{y}^{*}\right) a-F}{a(1-F)+F}>-\frac{(1-F)\left(\bar{E}-\hat{y}^{*}\right)}{E} .
$$

Therefore, whether or not (28) holds depends on the parameters of the model and the distribution function. A sufficient condition for this to hold is that

$$
\frac{f\left(\bar{E}-\hat{y}^{*}\right) a-F}{a(1-F)+F}>0
$$

which can be simplified to

$$
a f\left(\bar{E}-\hat{y}^{*}\right)>F
$$

For a given distribution function, this condition is more likely to be satisfied for large $a$. If (28) holds for $a=1$ then (due to $\hat{y}^{*}=E$ in this case), median income $y^{M}$ is below the equilibrium cutoff both before and after the change in $\bar{E}$. If Assumption 1 is satisfied and hence the median earner is the decisive voter, the demand for redistribution will increase by more 
than in the unbiased case if $\frac{y^{M}}{E_{p}}$ decreases by more than $\frac{y^{M}}{E}$.

If $\underline{E}$ decreases and $\bar{E}$ increases at the same time, the change in the equilibrium cutoff is given by

$$
d \hat{y}^{*}=\frac{F \Delta \underline{E}+a(1-F) \Delta \bar{E}}{a(1-F)+F}
$$

and hence whether $\hat{y}^{*}$ increases or decreases depends on the sign of $F \Delta \underline{E}+$ $a(1-F) \Delta \bar{E}$. If $a(1-F) \bar{E}+F \underline{E}$ decreases ${ }^{27}, \hat{y}^{*}$ goes down. The change in the perceived equality ratio $\frac{y^{M}}{E_{p}}$ amounts to

$$
d\left(\frac{y^{M}}{E_{p}}\right)=\frac{d y^{M} E_{p}-y^{M} d E_{p}}{\left(E_{p}\right)^{2}}=\left(\frac{\Delta y^{M}}{y^{M}}-\frac{d E_{p}}{E_{p}}\right) \frac{y^{M}}{E_{p}}
$$

where

$$
E_{p}=F \underline{E}+(1-F) \bar{E}_{p} .
$$

If both $\bar{E}$ and $\underline{E}$ change, then

$$
\begin{gathered}
d E_{p}=F \Delta \underline{E}+\left(f \underline{E}+F \frac{\partial \underline{E}}{\partial \hat{y}}\right) d \hat{y}+(1-F) d \bar{E}_{p}-f \bar{E}_{p} d \hat{y} \\
=F \Delta \underline{E}+(1-F)(1-\beta(1-F)) \Delta \bar{E}+(1-F) \beta[(1-F)+f(\bar{E}-\hat{y})] d \hat{y}^{*}
\end{gathered}
$$

Suppose that $F \Delta \underline{E}+a(1-F) \Delta \bar{E}$ is negative but $F \Delta \underline{E}+(1-F) \Delta \bar{E}$ is positive (implying that $a<1$ ), such that $\hat{y}^{*}$ decreases due to an increase in $\bar{E}$ and a decrease in $\underline{E}$, and average income $E$ increases. Suppose also that $y^{M}$ decreases. Then the true equality ratio decreases, because the numerator decreases and the denominator increases:

$$
d\left(\frac{y^{M}}{E}\right)=\left(\frac{\Delta y^{M}}{y^{M}}-\frac{\Delta E}{E}\right) \frac{y^{M}}{E}<0
$$

The change in the perceived equality ratio is given by

$$
d\left(\frac{y^{M}}{E_{p}}\right)=\left(\frac{\Delta y^{M}}{y^{M}}-\frac{d E_{p}}{E_{p}}\right) \frac{y^{M}}{E_{p}}
$$

\footnotetext{
${ }^{27}$ Note that if $a<1$ (i.e. the poor are less naive than the rich) I can have that $a(1-F) \bar{E}+F \underline{E}$ decreases, while $E=(1-F) \bar{E}+F \underline{E}$ stays constant. An increase in inequality while $E$ stays constant is probably the closest to reality that this model can get, as I have not modelled growth here. If I would have modelled growth, then this increase in inequality where $\underline{E}$ decreases and $\bar{E}$ increases while $E$ stays constant would translate to $\underline{E}$ constant and $\bar{E}$ increasing while $E$ increases, which is probably what has happened over the last 30 years in the US and Europe. I have refrained from modelling growth here, because this would just have complicated the analysis $\left(\hat{y}^{*}\right.$ would have a time trend etc.) while not changing the results about existence, uniqueness etc.
} 
(and assume that Assumption 1 holds before and after the change, such that the perceived equality ratio determines redistribution). (30) implies that $E_{p}$ will increase by less than $E$. The percentage decline in the perceived equality ratio, $\frac{\Delta y^{M}}{y^{M}}-\frac{d E_{p}}{E_{p}}$ can therefore - for certain changes $F \Delta \underline{E}+a(1-$ $F) \Delta \bar{E}<0$ (but $F \Delta \underline{E}+(1-F) \Delta \bar{E}>0$ ) - be smaller than the percentage decline in the true equality ratio, $\frac{d y^{M}}{y^{M}}-\frac{\Delta E}{E}$. Moreover, the change in the shape of the income distribution can be such that $d\left(\frac{y^{M}}{E_{p}}\right)$ is positive, and hence the demand for redistribution can go down as inequality increases.

If $a=1$ then the equilibrium cutoff goes down if average income decreases and goes up if average income increases due to the change in inequality. If $\Delta \bar{E}=-\Delta \underline{E}$ then $E$ decreases iff $F(E)>1-F(E)$, i.e. if the income distribution is positively skewed. In that case, the percentage decrease in the perceived equality ratio is smaller (in absolute value) than the percentage decrease in the true equality ratio iff

$$
\begin{gathered}
\left(\frac{\Delta y^{M}}{y^{M}}-\frac{d E_{p}}{E_{p}}\right)>\left(\frac{\Delta y^{M}}{y^{M}}-\frac{\Delta E}{E}\right) \\
\Longleftrightarrow \frac{d E_{p}}{E_{p}}<\frac{\Delta E}{E} \\
\Longleftrightarrow \frac{F \Delta \underline{E}+(1-F)(1-\beta(1-F)) \Delta \bar{E}+(1-F) \beta[(1-F)+f(\bar{E}-\hat{y})] \Delta E}{E-\beta(1-F)^{2}(\bar{E}-E)}<\frac{\Delta E}{E} \\
\Longleftrightarrow \frac{\Delta E-\beta(1-F)^{2} \Delta \bar{E}+(1-F) \beta[(1-F)+f(\bar{E}-\hat{y})] \Delta E}{E-\beta(1-F)^{2}(\bar{E}-E)}<\frac{\Delta E}{E} \\
\Longleftrightarrow \frac{1-\beta(1-F)^{2} \frac{\Delta \bar{E}}{\Delta E}+(1-F) \beta[(1-F)+f(\bar{E}-\hat{y})]}{E-\beta(1-F)^{2}(\bar{E}-E)}>\frac{1}{E} \\
\qquad E-\beta(1-F)^{2} \frac{\Delta \bar{E}}{\Delta E} E+(1-F) \beta[(1-F)+f(\bar{E}-\hat{y})] E>E-\beta(1-F)^{2}(\bar{E}-E) \\
\Longleftrightarrow \frac{(\bar{E}-E)}{E}+\left[1+\frac{f(\bar{E}-\hat{y})}{(1-F)}>\frac{\Delta \bar{E}}{\Delta E}\right.
\end{gathered}
$$

This inequality always holds, because the fraction on the RHS is negative. Hence, this type of increase in inequality always leads to a smaller increase in demand for redistribution if people are biased, compared to the unbiased case.

Conclusion 6 The effect of increasing inequality on support for redistribution if society is already segregated depends on the nature of the increase in inequality and on the rich and the poor's relative degree of naivety (resp. on a).

- If $a=1$ and $\underline{E}\left(\hat{y}^{*}\right)$ decreases ceteris paribus, then the equilibrium 
cutoff will go down. This leads to a change in the composition of the two groups in society, and, because the poor group is getting smaller, to an increase in poor people's bias - $\frac{E_{p}}{E}$ will decrease. As described above, this means that even though people in the poor group have become poorer relative to the rich, because they misperceive average income more after the change in inequality, their perceived equality ratio might not have decreased by much, or might even have increased. Hence, whether support for redistribution increases or decreases in this case depends on the poor's degree of naivety and on how much the median income decreases due to the increase in inequality. In any case, even if the change in inequality is such that the demand for redistribution increases, the increase is smaller than what would be expected in the framework of the Meltzer-Richard Model.

- If $a=1$ and $\bar{E}\left(\hat{y}^{*}\right)$ increases ceteris paribus, then the equilibrium cutoff will go up. This leads to a change in the composition of the two groups in society, and, because the poor group is getting larger, to a decrease in poor people's bias - $\frac{E_{p}}{E}$ will increase. However, if the income distribution is sufficiently unequal such that the median earner is the decisive voter, the median voter's preferred tax rate will still be smaller than in the absence of segregation and misperceptions. But the observed increase in support for redistribution might be larger if people are biased compared to a standard model without misperceptions, because as $\bar{E}\left(\hat{y}^{*}\right)$ increases demand for redistribution increases for two reasons: the median voter is getting poorer relative to the average, and the median voter is becoming less biased and hence more aware of the prevailing inequality. While the first effect is larger if people are unbiased, the second effect is only present if people are biased, and together, the two effects might lead to a larger increase than in the absence of a bias.

- If $a=1$ and both $\underline{E}\left(\hat{y}^{*}\right)$ decreases and $\bar{E}\left(\hat{y}^{*}\right)$ increases, the change in support for redistribution depends on whether the equilibrium cutoff increases or decreases. If $\Delta \bar{E}=-\Delta \underline{E}$, the equilibrium cutoff decreases if the income distribution is positively skewed. In this case the increase in support for redistribution will again be smaller than in the absence of misperceptions and we might even observe a decrease in support for redistribution.

Remark 1 I do not account for growth in my model, but my analysis would work in the same way if all variables would grow at a constant rate. In a model with growth, the case of $\bar{E}$ increasing and $\underline{E}$ decreasing would be translated into a situation where $\bar{E}$ increases a lot, while $\underline{E}$ stays constant (or increases only by a small rate), and we would see a decrease in the 
size of the poor group (corresponding to a decline in $\hat{y}^{*}$ with zero growth) if the distribution is sufficiently positively skewed. As Saez and Zucman (2016) point out, this constellation of high income growth of the rich accompanied by negligible growth rates of the bottom percentiles of the income distribution, is exactly what occurred during the past decades (at least in the US). Hence, my model can explain why, while inequality was increasing in the US over the past decades, people were, at least in the beginning, not demanding higher redistribution rates in response (if anything, then they were in favour of lower redistribution rates, as documented by Kuziemko et al. (2015), who analyze the evolution of preferences for redistribution in the General Social Survey (GSS)).

\section{Appendix B: Empirical Appendix}

\subsection{Working with Amazon Mechanical Turk}

For tax reasons, it is not possible for researchers living outside the United States to use Amazon Mechanical Turk directly. Therefore, I used the Amazon requester MTurkData to publish my survey via Amazon Mechanical Turk. They check the survey for compliance with Amazon's Terms and Conditions, publish it on MTurk and deal with the payment of the workers afterwards.

The advantages and disadvantages of working with Amazon Mechanical Turk have been discussed by Kuziemko et al (2015) in their online appendix. I agree with them that a major advantage of using MTurk is the speed of gathering responses: In my case, it took less than two hours to get 600 responses. There might in general be doubts about the quality of the responses, but it is possible to screen the MTurk workers based on their ratings for previous tasks. Using MTurk is also relatively cheap, as researchers design the survey themselves, instead of having it designed by a professional survey company. (Note also that I did not keep costs low at the expense of the respondents: they were all paid an hourly wage of 9 dollars.) One disadvantage of using MTurk is definitely that the obtained sample is usually not as representative as other, more expensive, online panel surveys (see below for a description of my own sample). However, as long as one keeps this in mind when interpreting the results, I think this is tolerable, especially when working with respondents from the United States, where MTurk is relatively well known and the pool of workers is therefore fairly representative. 


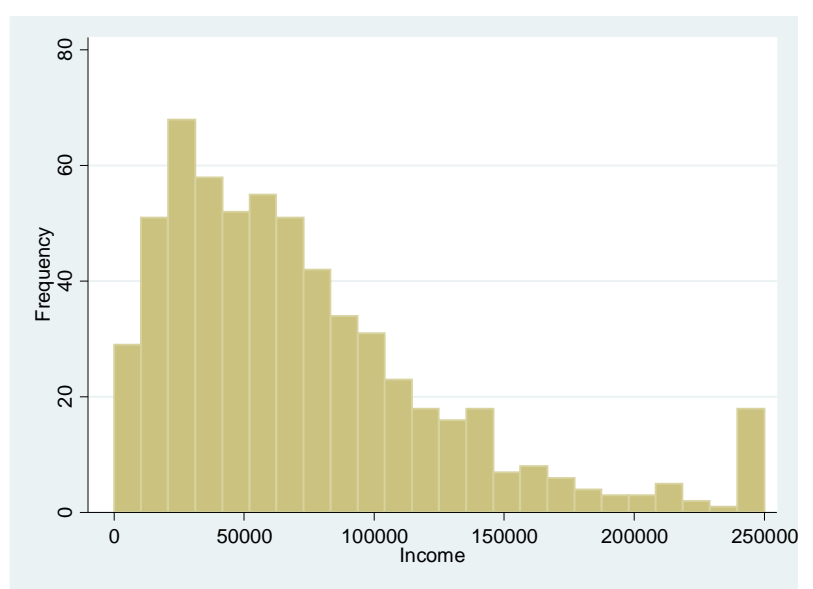

Figure 5: Sample household income distribution

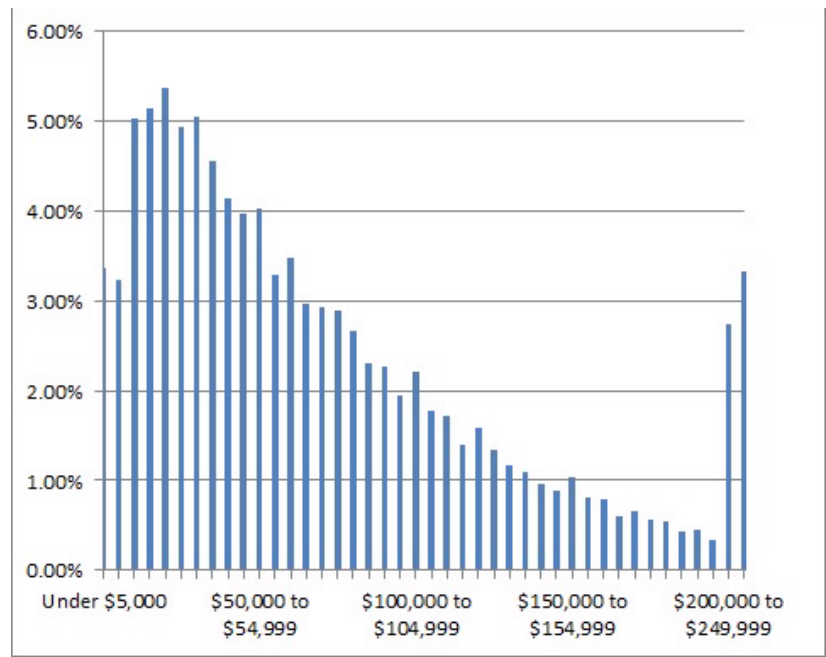

Figure 6: US household income distribution 2015 (Source: US Census Current Population Survey)

\subsection{Sample characteristics}

The sample is $83 \%$ White, $8.3 \%$ Black, $5.3 \%$ Asian and $1.5 \%$ Native American (the rest is "of other ethnicity"). Average age is $36.78,44 \%$ of respondents are married. $68 \%$ are full- or part-time employees, $17 \%$ are self-employed and $13 \%$ are unemployed or not in the labour force. The respondents are very well educated, $63 \%$ have completed some kind of college degree. Hence, compared to other (more representative and commonly used) online panel surveys cited in the online appendix of Kuziemko et al. my sample is younger, more educated and has fewer minorities. The household income distribution of the sample is roughly similar to the actual US household distribution (see Figures 5 and 6). 


\subsubsection{Social Segregation: Description of Factor Analysis}

In the survey, I ask several questions about people's colleagues at work, friends and family (spouse and siblings, if applicable). This is an attempt at identifying how diverse a person's social circle is. The underlying hypothesis is that an individual is more "socially segregated" the more homogenous and similar to herself her social circle is. However, it turned out that some of the questions were practically useless for my analysis in this relatively small sample: As less than half of the respondents are married, it turned out that using spouse characteristics to categorize social segregation would exclude a big part of the sample, and a similar reason can be applied to sibling characteristics. I therefore decided to exclude those variables from my factor analysis. Furthermore, I excluded variables indicating whether friends or colleagues have the same mother tongue, because I figured out that these variables predominantly serve to identify Hispanics in the sample and do not provide much variation. Hence, the factor analysis utilizes four categorical variables classifying the similarity of friends' and colleagues' education and income level. The variables take on the value 0 if the respondent has answered that all of their friends/ colleagues are different to them in the respective area (e.g. the variable friends_educ is 0 if the respondent states that all of her friends have a different education level than herself) and is then increasing in the degree of similarity (i.e. 1 if most friends have different education levels,... up to 4 if all friends have the same education level as the respondent). Hence, the higher the value of each categorical variable, the higher the respondent's degree of social segregation.

The results of the factor analysis are presented in the main text. 